\title{
Structure of level sets and Sard-type properties of Lipschitz maps
}

\author{
Giovanni Alberti, Stefano Bianchini and Gianluca Crippa
}

\begin{abstract}
We consider certain properties of maps of class $C^{2}$ from $\mathbb{R}^{d}$ to $\mathbb{R}^{d-1}$ that are strictly related to Sard's theorem, and we show that some of them can be extended to Lipschitz maps, while others require some additional regularity. We also give examples showing that, in terms of regularity, our results are optimal.
\end{abstract}

Mathematics Subject Classification (2010): 26B35 (primary); 26B10, 26B05, 49Q15, 58C25 (secondary).

\section{Introduction}

In this paper we study three problems which are strictly interconnected and ultimately related to Sard's theorem: structure of the level sets of maps from $\mathbb{R}^{d}$ to $\mathbb{R}^{d-1}$, weak Sard property of functions from $\mathbb{R}^{2}$ to $\mathbb{R}$, and locality of the divergence operator. Some of the questions we consider originated from a PDE problem studied in the companion paper [1]; they are however interesting in their own right, and this paper is in fact largely independent of [1].

Structure of level sets. In case of maps $f: \mathbb{R}^{d} \rightarrow \mathbb{R}^{d-1}$ of class $C^{2}$, Sard's theorem (see [17] and [12, Chapter 3, Theorem 1.3]) ${ }^{1}$ states that the set of critical values of $f$, namely the image according to $f$ of the critical set

$$
S:=\{x: \operatorname{rank}(\nabla f(x))<d-1\},
$$

has (Lebesgue) measure zero. By the Implicit Function Theorem, this property implies the following structure result: for a.e. $y \in \mathbb{R}^{d-1}$ the connected components of the level set $E_{y}:=f^{-1}(y)$ are simple curves (of class $C^{2}$ ).

This work has been partially supported by the italian Ministry of Education, University and Research (MIUR) through the 2006 PRIN Grant "Metodi variazionali nella teoria del trasporto ottimo di massa e nella teoria geometrica della misura", and by the European Research Council through the 2009 Starting Grant "Hyperbolic Systems of Conservation Laws: singular limits, properties of solutions and control problems".

Received July 20, 2011; accepted December 12, 2011.

${ }^{1}$ For more general formulations see also [9, Theorem 3.4.3] and $[2,3,6]$. 
For Sard's theorem to hold, the regularity assumption on $f$ can be variously weakened, but in any case $f$ must be at least twice differentiable in the Sobolev sense. ${ }^{2}$ However, a variant of the structure theorem holds even with lower regularity: in Theorem 2.5 , statement (iv), we show that if $f: \mathbb{R}^{2} \rightarrow \mathbb{R}$ is Lipschitz, then for a.e. $y \in \mathbb{R}$ the connected components of $E_{y}$ are either simple curves or consist of single points, and in statement (v) we show that the same holds if $d \geq 3$ and $f: \mathbb{R}^{d} \rightarrow \mathbb{R}^{d-1}$ is a map of class $C^{1,1 / 2}$.

Note that for $d \geq 3$ it is not enough to assume that $f$ is Lipschitz: in Section 3 we construct examples of maps $f: \mathbb{R}^{d} \rightarrow \mathbb{R}^{d-1}$ of class $C^{1, \alpha}$ for every $d \geq 3$ and $\alpha<1 /(d-1)$ such that for an open set of values $y$ the level set $E_{y}$ contains a Y-shaped subset, or triod (see Subsection 2.3 for a precise definition), and therefore at least one connected component of $E_{y}$ is neither a point nor a simple curve.

Weak Sard property. Given a function $f: \mathbb{R}^{2} \rightarrow \mathbb{R}$ of class $C^{2}$, consider the measure $\mu$ on $\mathbb{R}$ given by the push-forward according to $f$ of the restriction of Lebesgue measure to the critical set $S$ defined in (1.1). The measure $\mu$ is supported on the set $f(S)$, which is negligible by Sard's theorem, and therefore $\mu$ is singular with respect to the Lebesgue measure on $\mathbb{R}$ :

$$
\mu \perp \mathscr{L}^{1}
$$

Formula (1.2) can be viewed as a weak version of Sard's theorem, and holds under the assumption that $f$ is (locally) of class $W^{2,1}, c f$. [1, Section 2.15(v)].

In Section 4 we prove that the last assumption is essentially optimal; more precisely we construct a function $f: \mathbb{R}^{2} \rightarrow \mathbb{R}$ of class $C^{1, \alpha}$ for every $\alpha<1$ (and therefore also of class $W^{\beta, p}$ for every $\beta<2$ and $p \leq+\infty$ ) such that (1.2) does not hold.

We actually show that this function does not satisfy an even weaker version of (1.2), called weak Sard property, where in the definition of $\mu$ the critical set $S$ is replaced by $S \cap E^{*}$, with $E^{*}$ the union of all connected components with positive length of all level sets.

The relevance of the weak Sard property lies in the following result [1, Theorem 4.7]: let $b$ be a bounded divergence-free vector field on the plane, then the continuity equation $\partial_{t} u+\operatorname{div}(b u)=0$ admits a unique bounded solution for every bounded initial datum $u_{0}$ if and only if the potential $f$ associated to $b$, namely the Lipschitz function that satisfies $b=\nabla^{\perp} f,{ }^{3}$ satisfies the weak Sard property.

Locality of the divergence operator. It is well-known that given a function $u$ on $\mathbb{R}^{d}$ which is (locally) of class $W^{1,1}$, the (distributional) gradient $\nabla u$ vanishes a.e. on

${ }^{2}$ For Sard's Theorem for maps of class $C^{1,1}$ see [2], for maps in the Sobolev class $W^{2, p}$ see [4, 10]. The constructions in [11,19] and [9, Section 3.4.4] give counterexamples to Sard's Theorem of class $C^{1, \alpha}$ for every $\alpha<1$, and therefore also of class $W^{\beta, p}$ for every $\beta<2$ and $1 \leq p \leq \infty$. ${ }^{3}$ Given a vector $v=\left(v_{1}, v_{2}\right)$ we write $v^{\perp}:=\left(-v_{2}, v_{1}\right)$, and $v^{\top}:=\left(v_{2},-v_{1}\right)$. Thus $\nabla^{\perp}:=$ $\left(-\partial_{2}, \partial_{1}\right)$. If a vector field $b$ is bounded and divergence-free then $b^{\top}$ is bounded and curl-free, and therefore there exists a Lipschitz function $f$ such that $\nabla f=b^{\top}$, that is, $\nabla^{\perp} f=b$. 
every Borel set $E$ where $u$ takes a.e. a constant value. This property is summarized by saying that the gradient is strongly local for Sobolev functions; it follows immediately that every first-order differential operator, including the divergence, is strongly local for (first-order) Sobolev functions.

It is then natural to ask whether first-order differential operators are strongly local even on larger spaces.

In Section 5 we show that, somewhat surprisingly, the answer for the divergence operator is negative, ${ }^{4}$ and that in two dimensions this fact is strictly related to the (lack of) weak Sard property for Lipschitz functions (Remark 5.1). More precisely, we construct a bounded vector field $b$ on the plane whose (distributional) divergence belongs to $L^{\infty}$, is non-trivial, and is supported in the set where $b$ vanishes; we than use $b$ to construct another example of Lipschitz function $f: \mathbb{R}^{2} \rightarrow \mathbb{R}$ without the weak Sard property.

ACKNOWLEDGEMENTS. We thank Luděk Zajíček for pointing out reference [15] and David Preiss for reference [16].

\section{Structure of level sets of Lipschitz maps}

We begin by recalling some basic notation and definitions used through the entire paper, more specific definitions will be introduced when needed.

2.1. Basic notation. Through the rest of this paper, sets and functions are tacitly assumed to be Borel measurable, and measures are always defined on the appropriate Borel $\sigma$-algebra.

We write $a \wedge b$ and $a \vee b$ respectively for the minimum and the maximum of the real numbers $a, b .^{5}$

Given a subset $E$ of a metric space $X$, we write $1_{E}: X \rightarrow\{0,1\}$ for the characteristic function of $E, \operatorname{Int}(E)$ for the the interior of $E$, and, for every $r>0$, $\mathscr{I}_{r} E$ for the closed $r$-neighbourhood of $E$, that is,

$$
\mathscr{I}_{r} E:=\{x \in X: \operatorname{dist}(x, E) \leq r\} .
$$

The class $\mathscr{F}(X)$ of all non-empty, closed subsets of $X$ is endowed with the Hausdorff distance

$$
d_{H}\left(C, C^{\prime}\right):=\inf \left\{r \in[0,+\infty]: C \subset \mathscr{I}_{r} C^{\prime}, C^{\prime} \subset \mathscr{I}_{r} C\right\}
$$

4 This answers in the negative a question raised by L. Ambrosio; a simpler but less explicit example has been constructed by C. De Lellis and B. Kirchheim.

5 The symbol $\wedge$ is sometimes used for the exterior product; the difference is clear from the context. 
A function (or a map) defined on a closed set $E$ in $\mathbb{R}^{d}$ is of class $C^{k}$ if it admits an extension of class $C^{k}$ to some open neighbourhood of $E$, and is of class $C^{k, \alpha}$ with $0 \leq \alpha \leq 1$ if it is of class $C^{k}$ and the $k$-th derivative is Hölder continuous with exponent $\alpha$.

Given a measure $\mu$ on $X$ and a positive function $\rho$ on $X$ we denote by $\rho \cdot \mu$ the measure on $X$ defined by $[\rho \cdot \mu](A):=\int_{A} \rho d \mu$. Hence $1_{E} \cdot \mu$ is the restriction of $\mu$ to the set $E$.

Given a map $f: X \rightarrow X^{\prime}$ and a measure $\mu$ on $X$, the push-forward of $\mu$ according to $f$ is the measure $f_{\#} \mu$ on $X^{\prime}$ defined by $\left[f_{\#} \mu\right](A):=\mu\left(f^{-1}(A)\right)$ for every Borel set $A$ contained in $X^{\prime}$.

As usual, $\mathscr{L}^{d}$ is the Lebesgue measure on $\mathbb{R}^{d}$ while $\mathscr{H}^{d}$ the $d$-dimensional Hausdorff measure on every metric space - the usual $d$-dimensional volume for subsets of $d$-dimensional surfaces of class $C^{1}$ in some Euclidean space. The length of a set $E$ is just the 1-dimensional Hausdorff measure $\mathscr{H}^{1}(E)$. When the measure is not specified, it is assumed to be the Lebesgue measure.

A set $E$ in $\mathbb{R}^{d}$ is $k$-rectifiable if it can be covered, except for an $\mathscr{H}^{k}$-negligible subset, by countably many $k$-dimensional surfaces of class $C^{1}$.

2.2. Curves. A curve in $\mathbb{R}^{d}$ is the image $C$ of a continuous, non-constant path $\gamma:[a, b] \rightarrow \mathbb{R}^{d}$ (the parametrization of $C$ ); thus $C$ is a compact connected set that contains infinitely many points. We say that $C$ is simple if it admits a parametrization $\gamma$ that is injective, closed if $\gamma$ satisfies $\gamma(a)=\gamma(b)$; and closed and simple if $\gamma(a)=\gamma(b)$ and $\gamma$ is injective on $[a, b)$.

If $\gamma$ is a parametrization of $C$ of class $W^{1,1}$, then $\mathscr{H}^{1}(C) \leq\|\dot{\gamma}\|_{1}$ and the equality holds whenever $\gamma$ is injective. Moreover it is always possible to find a strictly increasing function $\sigma:\left[a^{\prime}, b^{\prime}\right] \rightarrow[a, b]$ such that $\gamma \circ \sigma$ is a Lipschitz parametrization which satisfies $\left|(\gamma \circ \sigma)^{\prime}\right|=1$ a.e.

For closed curves, it is sometimes convenient to identify the end points of the domain $[a, b]$. This quotient space is denoted by $[a, b]^{*}$, and endowed with the distance

$$
d(x, y):=|x-y| \wedge(b-a-|x-y|) .
$$

A set $E$ in $\mathbb{R}^{d}$ is path-connected if every couple of points $x, y \in E$ can be joined by a curve contained in $E$ (that is, $x, y$ agree with the end points $\gamma(a), \gamma(b)$ of the curve).

2.3. Triods. A (simple) triod in $\mathbb{R}^{d}$ is any set $Y$ given by the union of three curves with only one end point $y$ in common, which we call center of $Y$. More precisely

$$
Y=C_{1} \cup C_{2} \cup C_{3}
$$

where each $C_{i}$ is a curve in $\mathbb{R}^{d}$ parametrized by $\gamma_{i}:\left[a_{i}, b_{i}\right] \rightarrow C_{i}$ and $\gamma_{i}\left(a_{i}\right)=y$, and the sets $\gamma_{i}\left(\left(a_{i}, b_{i}\right]\right)$ are pairwise disjoint.

2.4. Lipschitz maps. Through this section $d, k$ are positive integers such that $0<$ $k<d$, and $f$ is a Lipschitz map from (a subset of) $\mathbb{R}^{d}$ to $\mathbb{R}^{d-k}$; we denote by $f_{i}$, $i=1, \ldots, d-k$, the components of $f$. 
For every $y \in \mathbb{R}^{d-k}$ we denote by $E_{y}:=f^{-1}(y)$ the corresponding level set of $f$, and by $\mathscr{C}_{y}$ the family of all connected components $C$ of $E_{y}$ such that $\mathscr{H}^{k}(C)>0 ;^{6} E_{y}^{*}$ is the union of all $C$ in $\mathscr{C}_{y}$, and $E^{*}$ is the union of all $E_{y}^{*}$ with $y \in \mathbb{R}^{d-k}$. Both $E_{y}^{*}$ and $E^{*}$ are Borel sets (Proposition 6.1).

By Rademacher's theorem $f$ is differentiable at almost every point, and at such points we define the Jacobian

$$
J:=\left[\operatorname{det}\left(\nabla f \cdot \nabla^{t} f\right)\right]^{1 / 2} .
$$

We denote by $S$ the set of all points $x \in \mathbb{R}^{d}$ where either $f$ is not differentiable or $J(x)=0$; note that $J(x) \neq 0$ if and only if the matrix $\nabla f(x)$ has rank $d-k$.

If $k=1$ and $x \notin S$ then there exists a unique unit vector $\tau=\tau(x)$ such that $\tau$ is orthogonal to $\nabla f_{i}$ for every $i$ and the sequence $\left(\nabla f_{1}, \ldots, \nabla f_{d-1}, \tau\right)$ is a positively oriented basis of $\mathbb{R}^{d}$.

We can now state the main result of this section. Statements (i) and (ii) are immediate consequences of the coarea formula and have been included for the sake of clearness. For $d=2$ and $k=1$, a variant of statement (iii) was proved in [16, Section 11] under the more general assumption that $f$ is a continuous $B V$ function. Even though we could not find statement (iv) elsewhere, the key observation behind its proof - namely that for a continuous function on the plane only countably many level sets contain triods - is well-known.

Theorem 2.5. Let $f: \mathbb{R}^{d} \rightarrow \mathbb{R}^{d-k}$ be a Lipschitz map with compact support. In the notation of the previous subsections, the following statements hold for almost every $y \in \mathbb{R}^{d-k}$ :

(i) the level set $E_{y}$ is $k$-rectifiable and $\mathscr{H}^{k}\left(E_{y}\right)<+\infty$;

(ii) $\mathscr{H}^{k}\left(E_{y} \cap S\right)=0$, which means that for $\mathscr{H}^{k}$-a.e. $x \in E_{y}$ the map $f$ is differentiable at $x$ and the matrix $\nabla f(x)$ has rank $d-k$; moreover the kernel of this matrix is the tangent space to $E_{y}$ at $x$;

(iii) the family $\mathscr{C}_{y}$ is countable and $\mathscr{H}^{k}\left(E_{y} \backslash E_{y}^{*}\right)=0$;

(iv) for $k=1$ and $d=2$ every connected component $C$ of $E_{y}$ is either a point or a closed simple curve with a Lipschitz parametrization $\gamma:[a, b]^{*} \rightarrow C$ which is injective and satisfies $\gamma(t) \notin S$ and $\dot{\gamma}(t)=\tau(\gamma(t))$ for a.e. $t$;

(v) the result in the previous statement can be extended to $k=1$ and $d \geq 3$ provided that $f$ is of class $C^{1,1 / 2}$.

Remark 2.6. (i) The assumption that $f$ is defined on $\mathbb{R}^{d}$ and has compact support was made for the sake of simplicity. Under more general assumptions, the results on the local properties of generic level sets (rectifiability and so on) are clearly

${ }^{6}$ Since the length of a connected set is larger than its diameter, for $k=1$ the connected components in $\mathscr{C}_{y}$ are just those that contain more than one point.

${ }^{7}$ If $d=2$ and $k=1$ then $J=|\nabla f|$ and $\tau$ is the counter-clockwise rotation by $90^{\circ}$ of $\nabla f /|\nabla f|$. 
the same, while the results concerning the global structure (notably statement (iv)) require some obvious modifications.

(ii) In statement (iv), the possibility that some connected components of $E_{y}$ are points cannot be ruled out. Indeed, for every $\alpha<1$, there are functions $f: \mathbb{R}^{2} \rightarrow$ $\mathbb{R}$ of class $C^{1, \alpha}$ such that the set $E_{y} \backslash E_{y}^{*}$ - namely the union of all connected components of $E_{y}$ which consist of single points - is not empty for an interval of values $y .^{8}$

(iii) In Section 3 we show that for every $d \geq 3$ and every $\alpha<1 /(d-1)$ there exist maps $f: \mathbb{R}^{d} \rightarrow \mathbb{R}^{d-1}$ of class $C^{1, \alpha}$ such that the level set $E_{y}$ contains a triod ( $c f$. Subsection 2.3) for an open set of values $y$, and therefore its connected components cannot be just points or simple curves. This shows that the exponent $1 / 2$ in the assumption $f \in C^{1,1 / 2}$ in statement (v) is optimal for $d=3$. However we believe that the exponent $1 / 2$ is not optimal for $d \geq 4$.

(iv) The fact that a connected component of $E_{y}$ is a simple curve with Lipschitz parametrization (statement (iv)) does not imply that it can be locally represented as the graph of a Lipschitz function: there exist functions $f$ on the plane of class $C^{1, \alpha}$ with $\alpha<1$ such that every level set contains a cusp.

(v) Even knowing that a connected component of $E_{y}$ is a simple curve, the existence of a parametrization whose velocity field agrees a.e. with $\tau$ ( $c f$. statement (iv)) is not as immediate as it may look, because $\tau$ and $E_{y}$ lack almost any regularity. Our proof relies on the fact that generic level sets of Lipschitz maps can be endowed with the structure of rectifiable currents without boundary.

(vi) One might wonder if something similar to statement (iv) holds at least for functions $f: \mathbb{R}^{d} \rightarrow \mathbb{R}$ with $d>2$. As shown below, the key points in the proof of statement (iv) are that a family of pairwise disjoint triods in the plane is countable, and that a connected set in the plane with finite length which contains no triods is a simple curve. A natural generalization of the notion of triod could be the following: a connected, compact set $E$ in $\mathbb{R}^{d}$ is a $d$-triod with center $y$ if, for every open ball $B$ which contains $y$, the set $\bar{B} \backslash E$ has at least three connected components which intersect $\partial B$. However, even if it is still true that a family of pairwise disjoint $d$-triods in $\mathbb{R}^{d}$ is countable, very little can be said on the topological structure of $d$-triod-free connected sets with finite $\mathscr{H}^{d-1}$ measure.

The rest of this section is devoted to the proof of Theorem 2.5.

2.7. Coarea formula. The coarea formula (see e.g. [9, Section 3.2.11], [14, Corollary 5.2.6], or [18, Section 10]) states that for every Lipschitz map $f: \mathbb{R}^{d} \rightarrow \mathbb{R}^{d-k}$

8 Simple examples can be obtained by modifying the construction in [11]. Moreover, since the level sets $E_{y}$ of any continuous function contain isolated points only for countably many $y$, it turns out that $E_{y} \backslash E_{y}^{*}$ has the cardinality of continuum for a set of positive measure of $y$. An example of Lipschitz function on the plane such that the set $E_{y} \backslash E_{y}^{*}$ has the cardinality of continuum for a.e. $y$ is also given in [13]. 
and every positive Borel function $\phi: \mathbb{R}^{d} \rightarrow[0,+\infty]$ there holds

$$
\int_{\mathbb{R}^{d}} \phi J d \mathscr{L}^{d}=\int_{\mathbb{R}^{d-k}}\left[\int_{E_{y}} \phi d \mathscr{H}^{k}\right] d \mathscr{L}^{d-k}(y)
$$

(quite obviously, (2.3) holds even for real-valued functions $\phi$ provided that the integral at the left-hand side makes sense, e.g., for $\phi \in L^{1}\left(\mathbb{R}^{d}\right)$ ).

The coarea formula has two immediate consequences:

(i) Let $\phi$ be the constant function 1: if $f$ has compact support then the integral at the left-hand side of (2.3) is finite, and therefore $\mathscr{H}^{k}\left(E_{y}\right)$, which is the value of the integral between square brackets at the right-hand side, is finite for a.e. $y \in$ $\mathbb{R}^{d-k}$.

(ii) If $\phi$ is the characteristic function of the set $S$ defined in Subsection 2.4, then identity (2.3) implies that for a.e. $y \in \mathbb{R}^{d-k}$ there holds $\mathscr{H}^{k}\left(E_{y} \cap S\right)=0$. This means that for $\mathscr{H}^{k}$-a.e. $x \in E_{y}$ the map $f$ is differentiable at $x$ and $J(x) \neq 0$, that is, $\nabla f(x)$ has rank $d-k$. Hence $E_{y}$ admits a $k$-dimensional tangent space at $x$, namely the kernel of the matrix $\nabla f(x)$. This implies that $E_{y}$ is $k$-rectifiable, cf. [9, Theorem 3.2.15] or [18, Theorem 10.4].

Proof of statements (i) and (ii) of Theorem 2.5. These statements are included in Subsection 2.7.

Statement (iii) of Theorem 2.5 will be obtained as a corollary of Lemmas 2.11, 2.12 , and 2.13. In the next three subsections we recall some definitions and a few results that will be used in the proofs of these lemmas.

2.8. Connected components. We recall here some basic facts about the connected components of a set $E$ in $\mathbb{R}^{d}$, or more generally in a metric space $X$; for more details see for instance [7, Chapter 6].

A connected component of $E$ is any element of the class of connected subsets of $E$ which is maximal with respect to inclusion. The connected components of $E$ are pairwise disjoint, closed in $E$, and cover $E$.

Assume now that $E$ is compact. Then each connected component $C$ agrees with the intersection of all subsets of $E$ that are closed and open in $E$ and contain C [7, Theorem 6.1.23].

Every set which is open and closed in $E$ can be written as $U \cap E$ where $U$ is an open subset of $X$ such that $\partial U \cap E=\varnothing$. ${ }^{9}$ Hence $C$ is the intersection of the closures of all open sets $U$ which contain $C$ and satisfy $\partial U \cap E=\varnothing$. Therefore, under the further assumption that $X$ is second countable (or, equivalently, separable), we can find a decreasing sequence $U_{n}$ of such sets, the intersection of whose closures is still $C$. $^{10}$

${ }^{9}$ If $D$ is open and closed in $E$, then $D$ and $E \backslash D$ are disjoint and closed in $X$, and since $X$ is a normal space there exist disjoint open sets $U, V$ such that $D \subset U$ and $E \backslash D \subset V$; it is then easy to check that $U$ meets all requirements.

10 Recall that in a second countable space every family $\mathscr{F}$ of closed sets admits a countable subfamily $\mathscr{F}$ such that the intersection of $\mathscr{F}$ and the intersection of $\mathscr{F}$ agree. 
2.9. Rectifiable currents. We recall here some basic definitions about currents; for further details see for instance [14, Chapter 7] or [18, Chapter 6] .

A $k$-dimensional current on $\mathbb{R}^{d}$ is a linear functional on the space of $k$-forms on $\mathbb{R}^{d}$ of class $C_{c}^{\infty}$. The boundary of a $k$-current $T$ is the $(k-1)$-dimensional current $\partial T$ defined by $\langle\partial T ; \omega\rangle:=\langle T ; d \omega\rangle$, where $d \omega$ is the exterior derivative of $\omega$. The mass of $T$, denoted by $\mathbb{M}(T)$, is the supremum of $\langle T ; \omega\rangle$ among all $k$-forms $\omega$ that satisfy $|\omega| \leq 1$ everywhere; $\mathbb{M}$ is clearly a norm on the space of currents with finite mass.

Let $E$ be a $k$-rectifiable set in $\mathbb{R}^{d}$. An orientation of $E$ is a map $\zeta$ that associates to $\mathscr{H}^{k}$-a.e. $x$ in $E$ a unit, simple $k$-vector that spans the approximate tangent space to $E$ at $x$; a multiplicity is any integer-valued function $m$ on $E$ which is locally summable with respect to $\mathscr{H}^{k}$. To every choice of $E, \zeta, m$ is canonically associated the $k$-dimensional current $[E, \zeta, m]$ defined by

$$
\langle[E, \zeta, m] ; \omega\rangle:=\int_{E}\langle\omega ; \zeta\rangle m d \mathscr{H}^{k}
$$

for every $k$-form $\omega$ of class $C_{c}^{\infty}$ on $\mathbb{R}^{d}$. Hence the mass of $[E, \zeta, m]$ is equal to $\int_{E}|m| d \mathscr{H}^{k}$. Currents of this type are called rectifiable. ${ }^{11}$

2.10. Current structure of level sets. Take $S$ as in Subsection 2.4. For every $x \in \mathbb{R}^{d} \backslash S$ the kernel of the matrix $\nabla f$ has dimension $k$, and therefore we can choose an orthonormal basis $\left\{\tau_{1}, \ldots, \tau_{k}\right\}$ such that $\left(\nabla f_{1}, \ldots, \nabla f_{d-k}, \tau_{1}, \ldots, \tau_{k}\right)$ is a positively oriented basis of $\mathbb{R}^{d}$; we denote by $\tau$ the simple $k$-vector $\tau_{1} \wedge \cdots \wedge \tau_{k}{ }^{12}$

Statement (ii) in Subsection 2.7 shows that for a.e. $y$ the $k$-vector field $\tau$ defines an orientation of $E_{y}$. We denote by $T_{y}$ the $k$-dimensional current associated with the set $E_{y}$, the orientation $\tau$, and constant multiplicity 1 , that is, $T_{y}:=\left[E_{y}, \tau, 1\right]$. that is

The essential fact about the current $T_{y}$ is that its boundary vanishes for a.e. $y$,

$$
\left\langle\partial T_{y} ; \omega\right\rangle:=\left\langle T_{y} ; d \omega\right\rangle:=\int_{E_{y}}\langle d \omega ; \tau\rangle d \mathscr{H}^{k}=0
$$

for every $(k-1)$-form $\omega$ of class $C_{c}^{\infty}$ on $\mathbb{R}^{d}$.

The proof goes as follows: the currents $T_{y}$ are the slices according to the map $f$ of the $d$-dimensional rectifiable current $T:=\left[\mathbb{R}^{d}, \zeta, 1\right]$, where $\zeta$ is the canonical orientation of $\mathbb{R}^{d}$. In general, the boundaries of the slices $T_{y}$ agree with the slices of the boundary $\partial T$ for a.e. $y$ (see [9, Section 4.3.1]), and since in this particular case $\partial T=0$, then $\partial T_{y}=0$ for a.e. $y$.

${ }^{11}$ Examples of $k$-dimensional rectifiable currents are obtained by taking a $k$-dimensional oriented surface $E$ of class $C^{1}$, endowed with constant multiplicity 1 . In this case the mass agrees with the $k$-dimensional volume of $E$, and the boundary of $E$ in the sense of currents agrees with the $(k-1)$-dimensional current associated to the usual boundary $\partial E$, endowed with the canonical orientation and constant multiplicity 1 .

12 Note that $\tau$ is is uniquely determined by the assumptions on $\tau_{1}, \ldots, \tau_{k}$, and for $k=1$ it agrees with the vector defined in Subsection 2.4. 
Lemma 2.11. Let $T:=[E, \zeta, m]$ be a rectifiable $k$-current in $\mathbb{R}^{d}$, and let $T^{\prime}:=$ $[E \cap A, \zeta, m]$ where $A$ is a set in $\mathbb{R}^{d}$. If $\partial T=0$ and the boundary of $A$ does not intersect the closure of $E$, then $\partial T^{\prime}=0$.

Proof. Since $\partial A \cap \bar{E}=\varnothing$, the sets $E \cap A$ and $E \backslash A$ have disjoint closures, and we can find a smooth function $\sigma: \mathbb{R}^{d} \rightarrow[0,1]$ that is equal to 1 on some neighbourhood of $E \cap A$, and to 0 on some neighbourhood of $E \backslash A$. Then for every form $\omega$ there holds

$$
\begin{aligned}
\left\langle\partial T^{\prime} ; \omega\right\rangle=\left\langle T^{\prime} ; d \omega\right\rangle=\langle T ; \sigma d \omega\rangle & =\langle T ; \sigma d \omega+d \sigma \wedge \omega\rangle \\
& =\langle T ; d(\sigma \omega)\rangle=\langle\partial T ; \sigma \omega\rangle=0
\end{aligned}
$$

(the second identity follows by the definition of $T^{\prime}$ and the fact that $\sigma=1$ on $E \cap A$ and $\sigma=0$ on $E \backslash A$. The third identity follows by the fact that $d \sigma=0$ on $E$ ).

Lemma 2.12. Let $T:=[E, \zeta, m]$ be a rectifiable $k$-current in $\mathbb{R}^{d}$ with $E$ bounded, and let $T^{\prime}:=[E \cap C, \zeta, m]$ where $C$ is a connected component of the closure of $E$. If $\partial T=0$ then $\partial T^{\prime}=0$.

Proof. Since $C$ is a connected component of $\bar{E}$, we can find a decreasing sequence of open sets $U_{n}$ such that the intersection is $C$ and $\partial U_{n} \cap \bar{E}=\varnothing$ for every $n$ (see Subsection 2.8). We set $T_{n}:=\left[E \cap U_{n}, \zeta, m\right]$. Hence Lemma 2.11 implies $\partial T_{n}=0$, and since $T_{n}$ converge to $T^{\prime}$ in the mass norm, then $\partial T_{n}$ converge to $\partial T^{\prime}$ in the weak topology of currents, and therefore $\partial T^{\prime}=0$.

Lemma 2.13. Let $E_{y}$ be a level set of $f$ such that $\mathscr{H}^{k}\left(E_{y}\right)<+\infty$ and the associated current $T_{y}$ is well-defined and has no boundary (cf. Subsection 2.10). Then $\mathscr{H}^{k}\left(E_{y} \backslash E_{y}^{*}\right)=0$.

Proof. Set $B:=E_{y} \backslash E_{y}^{*}$, fix $\delta>0$, and take an open set $A_{\delta}$ such that $B \subset A_{\delta}$ and

$$
\mathscr{H}^{k}\left(E_{y} \cap A_{\delta}\right) \leq \mathscr{H}^{k}(B)+\delta .
$$

Recall that $B$ is the union of the connected components of $E_{y}$ which are $\mathscr{H}^{k}$ negligible. For every such connected component $C$, we can find an open neighbourhood $U$ such that $C \subset U \subset A_{\delta}, \mathscr{H}^{k}\left(E_{y} \cap U\right) \leq \delta$, and $\partial U \cap E_{y}=\varnothing$ (see Subsection 2.8). From such family of neighbourhoods we extract a countable subfamily $\left\{U_{n}\right\}$ that covers $B$, and set $V_{n}:=U_{n} \backslash\left(U_{1} \cup \cdots \cup U_{n-1}\right)$ for every $n$. The sets $V_{n}$ are pairwise disjoint and cover $B$, and one easily checks that $\partial V_{n} \cap E_{y}=\varnothing$ for every $n$.

We then set $T_{n}:=\left[E_{y} \cap V_{n}, \tau, 1\right]$. Thus the sum $\sum_{n} T_{n}$ agrees with the current $T_{\delta}:=\left[E_{y} \cap V_{\delta}, \tau, 1\right]$ where $V_{\delta}$ is the union of the sets $V_{n}$. Moreover the properties of $U_{n}$ and $V_{n}$ yield $\mathbb{M}\left(T_{n}\right)=\mathscr{H}^{k}\left(E_{y} \cap V_{n}\right) \leq \mathscr{H}^{k}\left(E_{y} \cap U_{n}\right) \leq \delta$ and $\partial T_{n}=0$ (apply Lemma 2.11). Thus the isoperimetric theorem (see [14, Theorem 7.9.1], [18, Theorem 30.1]) yields $T_{n}=\partial S_{n}$ for some rectifiable $(k+1)$-current $S_{n}$ that satisfies

$$
\mathbb{M}\left(S_{n}\right) \leq c\left[\mathbb{M}\left(T_{n}\right)\right]^{1+1 / k} \leq c \mathbb{M}\left(T_{n}\right) \delta^{1 / k}=c \mathscr{H}^{k}\left(E_{y} \cap V_{n}\right) \delta^{1 / k},
$$


where $c$ is a constant that depends only on $d$ and $k$. Since the flat norm ${ }^{13}$ of $T_{n}$ satisfies $\mathbb{F}\left(T_{n}\right) \leq \mathbb{M}\left(S_{n}\right)$, the previous inequality yields

$$
\mathbb{F}\left(T_{n}\right) \leq c \mathscr{H}^{k}\left(E_{y} \cap V_{n}\right) \delta^{1 / k} .
$$

Taking the sum over all $n$ we obtain $\mathbb{F}\left(T_{\delta}\right) \leq c \mathscr{H}^{k}\left(E_{y}\right) \delta^{1 / k}$, and therefore $T_{\delta}$ tends to 0 with respect to the flat norm as $\delta \rightarrow 0$.

On the other hand, the inclusions $B \subset V_{\delta} \subset A_{\delta}$ and the choice of $A_{\delta}$ imply $\mathscr{H}^{k}\left(\left(E_{y} \cap V_{\delta}\right) \backslash B\right) \leq \delta$. Hence $T_{\delta}=\left[E_{y} \cap V_{\delta}, \tau, 1\right]$ converge to the current $[B, \tau, 1]$ with respect to the norm $\mathbb{M}$, and therefore also with respect to the flat norm $\mathbb{F}$ as $\delta \rightarrow 0$. Thus $[B, \tau, 1]$ must be equal to 0 , which means that $\mathscr{H}^{k}(B)=0$.

Proof of statement (iii) of Theorem 2.5. Let $A$ be the set of all $y$ such that statements (i) and (ii) of Theorem 2.5 hold and $T_{y}:=\left[E_{y}, \tau, 1\right]$ is a well-defined current without boundary ( $c f$. Subsection 2.10 ). Then $A$ has full measure in $\mathbb{R}^{d-k}$, and we claim that statement (iii) holds for every $y \in A$. Indeed, the elements of $\mathscr{C}_{y}$ are pairwise disjoint subsets of $E_{y}$ with positive $\mathscr{H}^{k}$-measure, and since $\mathscr{H}^{k}\left(E_{y}\right)$ is finite, $\mathscr{C}_{y}$ must be countable. Moreover $\mathscr{H}^{k}\left(E_{y} \backslash E_{y}^{*}\right)=0$ by Lemma 2.13.

Next we give some lemmas used in the proof of statements (iv) and (v) of Theorem 2.5.

Lemma 2.14. Let $T:=[C, \zeta, 1]$ be a 1-dimensional rectifiable current in $\mathbb{R}^{d}$, where $C$ is a curve with Lipschitz parametrization $\gamma:[a, b] \rightarrow C$ such that $|\dot{\gamma}|=$ 1 a.e. Assume that $\partial T=0$ and $C$ is simple. Then

(i) $C$ is closed;

(ii) either $\zeta \circ \gamma=\dot{\gamma}$ a.e. in $[a, b]$ or $\zeta \circ \gamma=-\dot{\gamma}$ a.e. in $[a, b]$.

Proof. Step 1. Since $\zeta(\gamma(t))$ and $\dot{\gamma}(t)$ are parallel unit vectors for a.e. $t$, there exists $\sigma:[a, b] \rightarrow\{ \pm 1\}$ such that $\zeta(\gamma(t))=\sigma(t) \dot{\gamma}(t)$ for a.e. $t$. Since $\gamma$ is injective at least on $[a, b)$, the assumption $\partial T=0$ can be re-written as

$$
0=\int_{C}\langle d \phi ; \zeta\rangle d \mathscr{H}^{1}=\int_{a}^{b}\langle d \phi \circ \gamma ; \dot{\gamma}\rangle \sigma d \mathscr{L}^{1}
$$

for every function (0-form) $\phi$ on $\mathbb{R}^{d}$ of class $C_{c}^{\infty}$. Since $\langle d \phi \circ \gamma ; \dot{\gamma}\rangle$ is the (distributional) derivative of $\phi \circ \gamma$, we obtain that

$$
0=\int_{a}^{b} \dot{\varphi} \sigma d \mathscr{L}^{1}
$$

for every test function $\varphi:[a, b] \rightarrow \mathbb{R}$ of the form $\varphi=\phi \circ \gamma$ where $\phi$ is a function on $\mathbb{R}^{d}$ of class $C_{c}^{\infty}$.

13 Here the flat norm $\mathbb{F}(T)$ of a current $T$ with compact support is the infimum of $\mathbb{M}(R)+\mathbb{M}(S)$ over all possible currents $R, S$ such that $T=R+\partial S, c f$. [9, Section 4.1.12]. 
Step 2: proof of statement (i). Assume by contradiction that $\gamma$ is injective on $[a, b]$, that is, $C$ is simple but not closed. Then Corollary 7.4 implies that equality (2.5) holds for all Lipschitz functions $\varphi:[a, b] \rightarrow \mathbb{R}$, and this allows us to conclude that $\sigma=0$ a.e., in contradiction with the fact that $\sigma= \pm 1$ a.e.

Step 3: proof of statement (ii). Since $C$ is simple, the parametrization $\gamma$ is injective on the interval with identified end points $[a, b]^{*}$ (cf. Subsection 2.2), and then Corollary 7.4 yields that (2.5) holds for all Lipschitz functions $\varphi:[a, b] \rightarrow \mathbb{R}$ such that $\varphi(a)=\varphi(b)$. This implies that the distributional derivative of $\sigma$ vanishes, and therefore either $\sigma=1$ a.e. or $\sigma=-1$ a.e., and the proof is concluded.

Lemma 2.15. Let $\mathscr{F}$ be a family of pairwise disjoint triods in $\mathbb{R}^{2}$. Then $\mathscr{F}$ is countable.

The above lemma has been proved in [15, Theorem 1]. For reader's convenience, we give a self-contained proof of this result in Section 8.

Lemma 2.16. Given a map $f: \mathbb{R}^{d} \rightarrow \mathbb{R}^{d-1}$, the following statements hold:

(i) if $d=2$, then the level set $E_{y}$ contains no triods for all $y \in \mathbb{R}$ except countably many; ${ }^{14}$

(ii) if $d \geq 3$ and $f$ is of class $C^{1,1 / 2}$, then the level set $E_{y}$ contains no triods for a.e. $y \in \mathbb{R}^{d-1}$.

Proof. Since the level sets of any map are pairwise disjoint, statement (i) follows immediately from Lemma 2.15 .

We prove statement (ii) by reduction to the case $d=2$. Consider the open set

$$
U:=\left\{x \in \mathbb{R}^{d}: \operatorname{rank}(\nabla f(x)) \geq d-2\right\} .
$$

Since $f$ is of class $C^{1,1 / 2}$, a refined version of Sard theorem [2, Theorem 2] shows that the level set $E_{y}$ is contained in $U$ for a.e. $y \in \mathbb{R}^{d-1}$, and therefore it is sufficient to prove statement (ii) for the restriction of $f$ to $U$.

Now, by applying the Implicit Function Theorem to $y=f(x)$ we can cover $U$ by open sets $V$ where $d-2$ of the variables $x_{i}$ can be written in terms of $d-2$ of the variables $y_{i}$; in other words, for every such $V$ there exists an open set $W$ in $\mathbb{R}^{d}$, a diffeomorphism $\psi: W \rightarrow V$ of class $C^{1}$, and a map $g: W \rightarrow \mathbb{R}$ such that, after a suitable re-numbering of the variables,

$$
f \circ \psi(t)=\left(t_{1}, \ldots, t_{d-2}, g(t)\right) \quad \text { for all } t \in W .
$$

Then it suffices to prove statement (ii) for the map $\tilde{f}:=f \circ \psi$.

Let $N$ be the set of all $y \in \mathbb{R}^{d-1}$ such that the level set $\tilde{E}_{y}$ of $\tilde{f}$ contains a triod, and for every $y^{\prime}=\left(y_{1}, \ldots, y_{d-2}\right) \in \mathbb{R}^{d-2}$ let $N_{y^{\prime}}$ be the set of all $y^{\prime \prime} \in \mathbb{R}$ such that $\left(y^{\prime}, y^{\prime \prime}\right) \in N$. Then statement (i) shows that $N_{y^{\prime}}$ is countable for every $y^{\prime}$, and therefore Fubini's Theorem implies that $N$ is negligible.

14 Note that $f$ does not need to be Lipschitz, and not even continuous. 
Lemma 2.17. Let $E$ be a closed, connected set in $\mathbb{R}^{d}$ with finite, strictly positive length. If E contains no triods, then it is a simple curve, possibly closed. More precisely, there exists a Lipschitz parametrization $\gamma:[a, b] \rightarrow E$ which is either injective on $[a, b]$ or satisfies $\gamma(a)=\gamma(b)$ and is injective on $[a, b)$.

Proof. Step 1. Recall the following well-known fact: a connected closed set $E$ with finite length is connected by simple curves. More precisely, for every $x, y$ in $E$ there exists an injective map $\gamma:[a, b] \rightarrow E$ such that $\gamma(a)=x, \gamma(b)=y$, and $\gamma$ is 1-Lipschitz, that is, it has Lipschitz constant at most 1 (see for instance [8, Lemma 3.12]).

Step 2. Let $\mathscr{F}$ be the family of all 1-Lipschitz maps $\gamma:[a, b] \rightarrow E$ (the domain $[a, b]$ may vary with $\gamma)$ that are injective on $(a, b)$. We order $\mathscr{F}$ by inclusion of graphs, that is, $\gamma_{1} \preceq \gamma_{2}$ if $\left[a_{1}, b_{1}\right] \subset\left[a_{2}, b_{2}\right]$ and $\gamma_{1}=\gamma_{2}$ on $\left[a_{1}, b_{1}\right]$. One easily checks that $\mathscr{F}$ admits a maximal element $\gamma:[a, b] \rightarrow E$. In the next steps we show that this is the parametrization we are looking for.

Step 3: either $\gamma$ is injective on $[a, b]$ or satisfies $\gamma(a)=\gamma(b)$ and is injective on $[a, b)$. Since $\gamma$ is injective on $(a, b)$, it suffices to show that for every $t \in(a, b)$ there holds $\gamma(t) \neq \gamma(a), \gamma(b)$. Assume by contradiction that $\gamma(t)=\gamma(a)$ for some $t$, and take a positive $\delta$ such that $a+2 \delta<t<b-\delta$. Then, contrary to the assumptions of the statement, $E$ contains the triod with center $y:=\gamma(a)=\gamma(t)$ given by the union of the following three curves: $C_{1}:=\gamma([a, a+\delta]), C_{2}:=$ $\gamma([t-\delta, t]), C_{3}:=\gamma([t, t+\delta])$ (see Figure 2.1(a)).
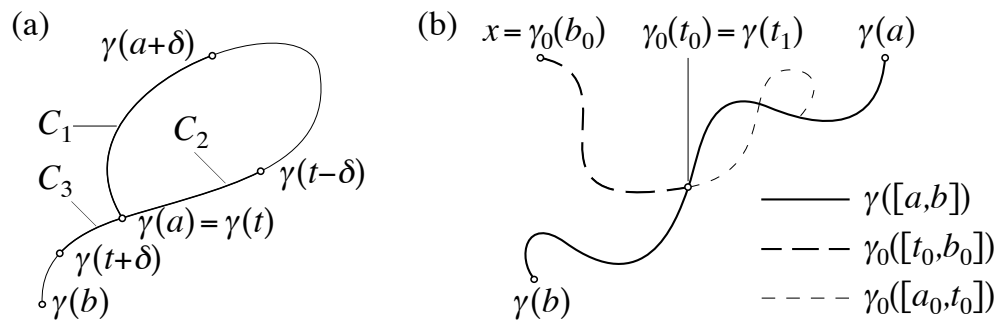

Figure 2.1. Construction of triods in the proof of Lemma 2.17.

Step 4: the image of $\gamma$ is $E$. Assume by contradiction that there exists $x \in E$ $\gamma([a, b])$. By Step 1 there exists an injective 1-Lipschitz map $\gamma_{0}:\left[a_{0}, b_{0}\right] \rightarrow E$ such that $\gamma_{0}\left(b_{0}\right)=x$ and $\gamma_{0}\left(a_{0}\right)$ is some point in $\gamma([a, b])$. Now, let $t_{0}$ be the largest of all $t \in\left[a_{0}, b_{0}\right]$ such that $\gamma_{0}(t) \in \gamma([a, b])$ and take $t_{1} \in[a, b]$ so that $\gamma\left(t_{1}\right)=\gamma_{0}\left(t_{0}\right)$.

If $\gamma$ is injective on $[a, b]$ - the other case is similar - we derive a contradiction in each of the following cases: i) $t_{1}=b$, ii) $t_{1}=a$, iii) $a<t_{1}<b$. If $t_{1}=b$, we extend the map $\gamma$ by setting $\gamma(t):=\gamma_{0}\left(t-b+t_{0}\right)$ for all $t \in\left[b, b+b_{0}-t_{0}\right]$ one easily checks that the extended map belongs to $\mathscr{F}$, in contradiction with the maximality of $\gamma$. A similar contradiction is obtained if $t_{1}=a$. Finally, if $a<t_{1}<$ $b$ then $E$ contains the triod with center $y:=\gamma\left(t_{1}\right)=\gamma_{0}\left(t_{0}\right)$ given by the union of 
the following three curves: $C_{1}:=\gamma_{0}\left(\left[t_{0}, b_{0}\right]\right), C_{2}:=\gamma\left(\left[a, t_{1}\right]\right), C_{3}:=\gamma\left(\left[t_{1}, b\right]\right)-$ see Figure 2.1(b).

Proof of statements (iv) and (v) of Theorem 2.5. Let $d=2$ or $d \geq 3$ and $f$ be of class $C^{1,1 / 2}$. Then for a.e. $y \in \mathbb{R}^{d-1}$ the level set $E_{y}$ has finite length (Subsection 2.7) and contains no triods (Lemma 2.16), and the associated current $T_{y}:=$ $\left[E_{y}, \tau, 1\right]$ is well-defined, rectifiable, and without boundary (Subsection 2.10). In particular the tangent vector $\tau$ is defined at $\mathscr{H}^{1}$-a.e. point of $E_{y}$.

For every such $y$, let $C$ be a connected component of $E_{y}$ which is not a point. Since $C$ contains no triods, Lemma 2.17 implies that $C$ is a simple curve with a Lipschitz parametrization $\gamma$, and we can further assume that $|\dot{\gamma}|=1$ a.e. The latter assumption implies that $\tau$ is defined at $\gamma(t)$ for a.e. $t$.

By Lemma 2.12 the current $T^{\prime}:=[C, \tau, 1]$ has no boundary, and therefore Lemma 2.14 implies that $C$ is a closed curve such that either $\tau \circ \gamma=\dot{\gamma}$ a.e. or $\tau \circ \gamma=-\dot{\gamma}$ a.e.; in the latter case we replace $\gamma(t)$ by $\gamma(-t)$ and the proof is concluded.

\section{Examples of maps with no triod-free level sets}

In this section we show that the assumption that $f$ is of class $C^{1,1 / 2}$ in statement (v) of Theorem 2.5 cannot be dropped. More precisely, given $d \geq 3$ and $\alpha<1 /(d-1)$, we construct a map $f$ of class $C^{1, \alpha}$ from $\mathbb{R}^{d}$ to a cube $Q_{0}$ in $\mathbb{R}^{d-1}$ such that every level set contains a triod, ${ }^{15}$ and therefore at least one of its connected components is neither a point nor a simple curve (Proposition 3.7(iii)).

This example shows that the Hölder exponent $1 / 2$ in Theorem 2.6(v) is optimal for $d=3$. As pointed out in Remark 2.6(iii), we believe the the optimal Hölder exponent for $d>3$ is the one suggested by this example, namely $1 /(d-1)$, and not $1 / 2$.

3.1. Idea of the construction. Assume for simplicity that $d=3$. The strategy for the construction of $f$ is roughly the following: we divide the target square $Q_{0}$ in a certain number $N$ of sub-squares $Q_{i}$ with side-length $\rho$, then we define $f$ on a cube $C_{0}$ minus $N$ disjoint sub-cubes $C_{i}$ with side-length $r$, so that the following key property holds: for every $y \in Q_{0}$ the level set $f^{-1}(y)$ contains three disjoint curves connecting three points $x_{1}, x_{2}, x_{3}$ on the boundary of $C_{0}$ with three points on the boundary of the cube $C_{i}$, where $i$ is chosen so that $y$ belongs to $Q_{i}$, see Figure 3.1(a). ${ }^{16}$

15 At first glance, this claim seems to contradict the fact that a generic level set of a Lipschitz map is a rectifiable current with multiplicity 1 and no boundary ( $c f$. Subsection 2.10). It is not so, since we do not claim that the level set coincides with the triod in a neighbourhood of the center of the triod.

16 This is the only part of this construction that requires more than two dimensions: as one can easily see in Figure 3.1, in two dimensions the joining curves for different values of $y$ would necessarily intersect, in contradiction with the fact that they are contained in different level sets. 
(a)

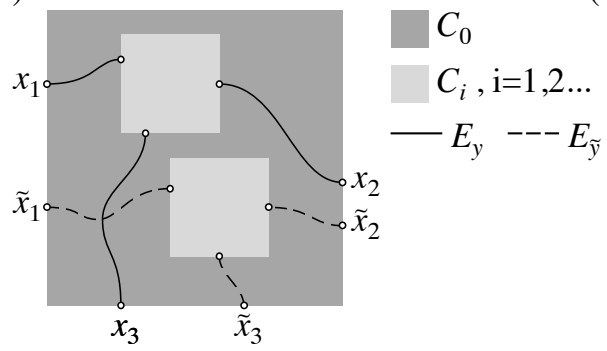

(b)

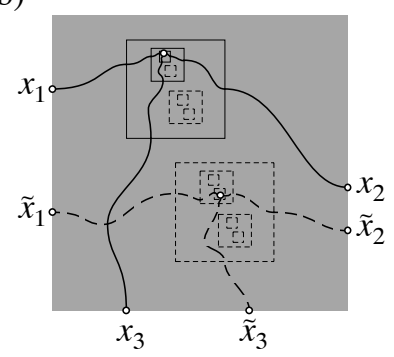

Figure 3.1. The components of the level sets $E_{y}$ and $E_{\tilde{y}}$ starting from the points $x_{j}$ and $\tilde{x}_{j}(j=1,2,3)$ after the first step of the construction (a), and at the end (b).

In the second step we replicate this construction within each $C_{i}$, so that $f$ takes values in $Q_{i}$, is defined outside $N$ sub-cubes with side-length $r^{2}$, and now $f^{-1}(y)$ contains disjoint curves connecting $x_{1}, x_{2}, x_{3}$ to three points on the boundary of one of these smaller cubes. And so on ...

As one can see from Figure 3.1(b), in the end each level set $E_{y}$ will contain three disjoint curves connecting $x_{1}, x_{2}, x_{3}$ to the same point, which means that $E_{y}$ contains a triod ( $c f$. Subsection 2.3).

Note that in the $(n+1)$-th step we define $f$ on each one of the $N^{n}$ cubes with side-length $r^{n}$ left over from the previous step minus $N$ sub-cubes with side-length $r^{n+1}$, so that each cube is mapped in a sub-square of $Q_{0}$ with side-length $\rho^{n}$. Thus the oscillation of $f$ on this cube is $\rho^{n}$, and this is enough to guarantee that in the end the map $f$ is continuous. As we shall see, if things are carefully arranged, $f$ turns out to be of class $C^{1, \alpha}$.

3.2. Notation. We denote the points in $\mathbb{R}^{d}$ by $x=\left(x^{\prime}, x_{d}\right)$ with $x^{\prime}:=\left(x_{1}, \ldots, x_{d-1}\right)$, and the points in $\mathbb{R}^{d-1}$ by the letter $y$. For every $x_{0} \in \mathbb{R}^{d}$ and every $\ell>0$, we denote by $C\left(x_{0}, \ell\right)$ the closed cube in $\mathbb{R}^{d}$ with center $x_{0}$ and side-length $\ell$ given by

$$
C\left(x_{0}, \ell\right):=x_{0}+\left[-\frac{\ell}{2}, \frac{\ell}{2}\right]^{d}
$$

We denote by $Q\left(y_{0}, \ell\right)$ the closed cube in $\mathbb{R}^{d-1}$, similarly defined.

Through this section we reserve the letter $C$ for $d$-dimensional closed cubes in $\mathbb{R}^{d}$ of the form $C\left(x_{0}, \ell\right)$, and the letter $Q$ for the $(d-1)$-dimensional closed cubes in $\mathbb{R}^{d-1}$ or in $\mathbb{R}^{d}$ with axes parallel to the coordinate axes. With the case $d=3$ in mind, we often refer to the former ones simply as "cubes" and to the latter ones as "squares".

We set

$$
C_{0}:=C(0,1)=\left[-\frac{1}{2}, \frac{1}{2}\right]^{d}, \quad Q_{0}:=Q(0,1)=\left[-\frac{1}{2}, \frac{1}{2}\right]^{d-1},
$$


and for every cube $C=C\left(x_{0}, \ell\right)$ we denote by $g_{C}$ the homothety on $\mathbb{R}^{d}$ which maps $C_{0}$ into $C$, that is,

$$
g_{C}(x):=x_{0}+\ell x \text { for all } x \in \mathbb{R}^{d} .
$$

Similarly, for every square $Q=Q\left(y_{0}, \ell\right)$ we set

$$
h_{Q}(y):=y_{0}+\ell y \quad \text { for all } y \in \mathbb{R}^{d-1} .
$$

3.3. A complete norm for $C^{k, \alpha}$. Given a real or vector-valued map $f$ defined on a subset $E$ of $\mathbb{R}^{d}$ and $\alpha \in(0,1]$, the homogeneous Hölder (semi-) norm of exponent $\alpha$ of $f$ is

$$
\|f\|_{C_{\text {hom }}^{0, \alpha}}:=\sup _{\substack{x, y \in E \\ x \neq y}} \frac{|f(x)-f(y)|}{|x-y|^{\alpha}} .
$$

Let be given an open set $A$ in $\mathbb{R}^{d}$, a point $x_{0} \in A$, and an integer $k \geq 0$. Among the many (equivalent) complete norms on the space $C^{k, \alpha}(A)$, the following is particularly convenient:

$$
\|f\|_{C^{k, \alpha}}:=\left\|\nabla^{k} f\right\|_{C_{\text {hom }}^{0, \alpha}}+\sum_{h=0}^{k}\left|\nabla^{h} f\left(x_{0}\right)\right| .
$$

The following interpolation inequality will be useful: if $E$ is a convex set with non-empty interior and $f$ is of class $C^{1}$ then $^{17}$

$$
\|f\|_{C_{\text {hom }}^{0, \alpha}} \leq 2\|f\|_{\infty}^{1-\alpha}\|\nabla f\|_{\infty}^{\alpha} .
$$

3.4. Construction of $C_{i}, Q_{i}, g_{i}, h_{i}$. For the rest of this section we fix a positive real number $\alpha$ such that

$$
\alpha<\frac{1}{d-1}
$$

We also fix an integer $N>1$ which is both a $d$-th and a $(d-1)$-th power of integers, e.g., $N=2^{d(d-1)}$. Since $N$ is a $(d-1)$-th power of an integer, we can cover the $(d-1)$-dimensional square $Q_{0}$ by $N$ squares $Q_{i}$ with pairwise disjoint interiors and side-length $\rho:=N^{-1 /(d-1)}$. Since $N$ is the $d$-th power of an integer, for every positive real number $r$ such that

$$
r<N^{-1 / d}
$$

we can find $N$ pairwise disjoint cubes $C_{i}$ with side-length $r$ contained in the interior of $C_{0}$. For every $i=1, \ldots, N$ we set

$$
g_{i}:=g_{C_{i}}, \quad h_{i}:=h_{Q_{i}}
$$

(thus $g_{i}$ and $h_{i}$ are homotheties with scaling factors $r$ and $\rho$, respectively).

${ }^{17}$ It suffices to estimate the numerator $|f(x)-f(y)|$ at the right-hand side of (3.1) by $2\|f\|_{\infty}$ when $|x-y| \geq\|f\|_{\infty}\|\nabla f\|_{\infty}^{-1}$, and by $\|\nabla f\|_{\infty}|x-y|$ otherwise. 
For the rest of this section we fix $r$ so that it satisfies (3.5) and

$$
\rho<r^{1+\alpha}
$$

This assumption is compatible with (3.5) because the upper bound on $\alpha$ and the definition of $\rho$ imply $\rho<\left(N^{-1 / d}\right)^{(1+\alpha)}$.

Finally, we define the open set

$$
A:=\operatorname{Int}\left(C_{0} \backslash\left(C_{1} \cup \cdots \cup C_{N}\right)\right) .
$$

3.5. Construction of $f_{0}$. We construct a map $f_{0}: \mathbb{R}^{d} \rightarrow Q_{0}$ with the following properties:

(i) $f_{0}$ is of class $C^{2}$ and has compact support;

(ii) $f_{0}$ agrees with $h_{i} \circ f_{0} \circ g_{i}^{-1}$ on a neighbourhood of $\partial C_{i}$ for $i=1, \ldots, N$;

(iii) there exists three pairwise disjoint sets $G_{j}$ contained in $\partial C_{0}$ such that the following holds: for every $i=1, \ldots, N$ and every $y \in Q_{i}$ both $f_{0}^{-1}(y) \cap G_{j}$ and $f_{0}^{-1}(y) \cap g_{i}\left(G_{j}\right)$ consist of single points, denoted by $x_{j}$ and $x_{j}^{\prime}$ respectively, and there exist pairwise disjoint curves joining $x_{j}$ and $x_{j}^{\prime}$ and contained in $f_{0}^{-1}(y) \cap A$ except for the end points, $c f$. Figure 3.1(a).

The construction of $f_{0}$ is divided in three steps.

Step 1 . For $j=1,2,3$ we define $G_{j}:=Q_{j}^{\prime} \times\{1 / 2\}$ where $Q_{1}^{\prime}, Q_{2}^{\prime}, Q_{3}^{\prime}$ are pairwise disjoint squares contained in $Q_{0}$. Then we choose $\varepsilon>0$ so that

(a) the $\varepsilon$-neighbourhoods $\mathscr{I}_{\varepsilon} G_{j}$ with $j=1,2,3$ are pairwise disjoint;

(b) $\mathscr{I}_{\varepsilon}\left(\partial C_{0}\right)$ and $\mathscr{I}_{r \varepsilon}\left(\partial C_{i}\right)$ are disjoint for $i=1, \ldots, N$ (see Figure 3.2(a)).

Then we set

$$
f_{0}(x):=h_{Q_{j}^{\prime}}^{-1}\left(x^{\prime}\right) \text { for every } x \in \mathscr{I}_{\varepsilon} G_{j},
$$

and take an arbitrary smooth extension of $f_{0}$ to the rest of $\mathscr{I}_{\varepsilon}\left(\partial C_{0}\right)$.

Step 2. We define $f_{0}$ on the sets $\mathscr{I}_{r \varepsilon}\left(\partial C_{i}\right)$ with $i=1, \ldots, N$ so that property (ii) above is satisfied, that is, $f_{0}:=h_{i} \circ f_{0} \circ g_{i}^{-1} \cdot 18$

Step 3. So far the map $f_{0}$ has been defined on a neighbourhood of the union of $\partial C_{i}$ with $i=0, \ldots, N$. Now we extend it to the rest of $\mathbb{R}^{d}$ so that property (iii) above is satisfied.

Let $y \in Q_{i}$ be fixed: by Step 1 there exists a unique point in $G_{j}$, denoted by $x_{j}(y)$, such that $f\left(x_{j}(y)\right)=y$, and by Step 2 there exists a unique point $x_{j}^{\prime}(y)$ in $g_{i}\left(G_{j}\right)$ such that $f\left(x_{j}^{\prime}(y)\right)=y$.

Roughly speaking, the idea is to choose for all $y \in Q_{0}$ and all $j=1,2,3$ pairwise disjoint curves joining $x_{j}(y)$ and $x_{j}^{\prime}(y)$ and contained in $A$ except the end

18 This definition is well-posed because the sets $\mathscr{I}_{\varepsilon}\left(\partial C_{0}\right)$ and $\mathscr{I}_{r \varepsilon}\left(\partial C_{i}\right)$ are pairwise disjoint. 
(a)

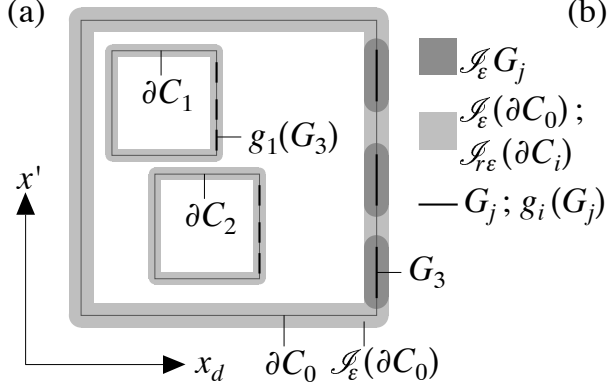

(b)

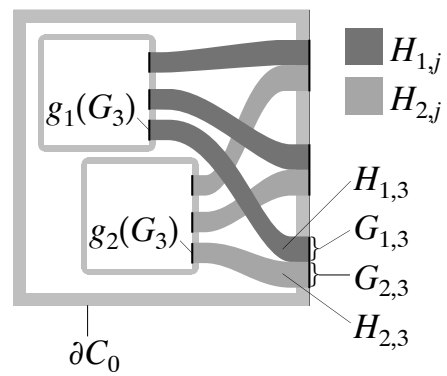

Figure 3.2. Sets involved in the construction of $f_{0}$ on $F_{1}(N=2)$ : contrary to what appears on the right, in dimension larger than two the sets $H_{i j}$ do not overlap.

points, and then set $f_{0}:=y$ on each curve. However, in order to get a smooth map we need a careful construction. For every $j=1,2,3$ and every $i=1, \ldots, N$, the sets

$$
G_{i j}:=G_{j} \cap f_{0}^{-1}\left(Q_{i}\right)
$$

are squares with pairwise disjoint interiors of the form $Q_{i j}^{\prime \prime} \times\{1 / 2\}$ which cover $G_{j}$, while the sets $g_{i}\left(G_{j}\right)$ are pairwise disjoint squares contained in $\partial C_{i}$ that can be written as $Q_{i j}^{\prime \prime} \times\left\{s_{i}\right\}$ for suitable $s_{i}$.

Therefore we can find closed sets $H_{i j}$, with pairwise disjoint interiors and contained in the closure of $A$, and diffeomorphisms

$$
\Psi_{i j}: Q_{0} \times[0,1] \rightarrow H_{i j}
$$

which map $Q_{0} \times\{0\}$ onto $g_{i}\left(G_{j}\right)$, and $Q_{0} \times\{1\}$ onto $G_{i j}$, see Figure 3.2(b), and more precisely

$$
\Psi_{i j}\left(x^{\prime}, t\right)= \begin{cases}\left(h_{Q_{i j}^{\prime \prime}}\left(x^{\prime}\right), s_{i}+t\right) & \text { for } x^{\prime} \in Q_{0}, t \in[0, r \varepsilon] \\ \left(h_{Q_{i j}^{\prime}}\left(x^{\prime}\right), t-1 / 2\right) & \text { for } x^{\prime} \in Q_{0}, t \in[1-\varepsilon, 1]\end{cases}
$$

By Step 1 and Step 2, the map $f_{0}$ is already defined on the points of $H_{i j}$ of the form $\Psi_{i j}\left(x^{\prime}, t\right)$ with $x^{\prime} \in Q_{0}$ and $t \in[0, r \varepsilon] \cup[1-\varepsilon, 1]$, and satisfies

$$
f_{0}\left(\Psi_{i j}\left(x^{\prime}, t\right)\right)=h_{i}^{-1}\left(x^{\prime}\right)
$$

Then we extend $f_{0}$ to the rest of $H_{i j}$ just by setting $f_{0}\left(\Psi_{i j}\left(x^{\prime}, t\right)\right):=h_{i}^{-1}\left(x^{\prime}\right)$ for every $x^{\prime} \in Q_{0}$ and every $t \in[0,1]$.

Finally we take an arbitrary extension with compact support of $f_{0}$ to the rest of $\mathbb{R}^{d} \cdot 19$

19 Such an extension can be taken of class $C^{2}$, and even smooth, provided that the diffeomorphisms $\Psi_{i j}$ are carefully chosen (we omit the verification). 
3.6. Construction of $f_{n}$. We denote by $J$ the set of indexes $\{1, \ldots, N\}$, and for every $n=2,3, \ldots$ and every $\mathbf{i}=\left(i_{1}, \ldots, i_{n}\right) \in J^{n}$ we define

$$
\begin{aligned}
& g_{\mathbf{i}}:=g_{i_{n}} \circ g_{i_{n-1}} \circ \cdots \circ g_{i_{1}}, \quad C_{\mathbf{i}}:=g_{\mathbf{i}}\left(C_{0}\right) \\
& h_{\mathbf{i}}:=h_{i_{n}} \circ h_{i_{n-1}} \circ \cdots \circ h_{i_{1}}, \quad Q_{\mathbf{i}}:=h_{\mathbf{i}}\left(Q_{0}\right)
\end{aligned}
$$

and

$$
A_{n}:=\mathbb{R}^{d} \backslash\left(\bigcup_{\mathbf{i} \in J^{n}} C_{\mathbf{i}}\right)
$$

For every $n=1,2, \ldots$ we define the map $f_{n}: \mathbb{R}^{d} \rightarrow Q_{0}$ by the recursive relation

$$
f_{n}(x):= \begin{cases}f_{n-1}(x) & \text { if } x \in A_{n} \\ h_{\mathbf{i}} \circ f_{0} \circ g_{\mathbf{i}}^{-1}(x) & \text { if } x \in C_{\mathbf{i}} \text { with } \mathbf{i} \in J^{n}\end{cases}
$$

Proposition 3.7. Take $\alpha$ and $f_{n}$ as in Subsection 3.4 and Subsection 3.6. Then

(i) the maps $f_{n}: \mathbb{R}^{d} \rightarrow Q_{n}$ are of class $C^{2}$;

(ii) the maps $f_{n}$ converge to a limit map $f: \mathbb{R}^{d} \rightarrow Q_{0}$ of class $C^{1, \alpha}$;

(iii) for every $y \in Q_{0}$ the level set $E_{y}:=f^{-1}(y)$ contains a triod, and therefore at least one of its connected component is neither a point nor a simple curve.

Proof. We prove statement (i) by induction on $n$. The map $f_{0}$ is of class $C^{2}$ by construction (property (i) in Subsection 3.5), and if $f_{n-1}$ is of class $C^{2}$ then formula (3.8) implies that $f_{n}$ is of class $C^{2}$ provided that $f_{n-1}$ agrees with $h_{\mathbf{i}} \circ f_{0} \circ g_{\mathbf{i}}^{-1}$ on a neighbourhood of $\partial C_{\mathbf{i}}$ for every $\mathbf{i} \in J^{n}$.

For every $\mathbf{i}=\left(i_{1}, \ldots, i_{n}\right) \in J^{n}$ let $\mathbf{i}^{\prime}=\left(i_{1}, \ldots, i_{n-1}\right)$. Formula (3.8) implies that $f_{n-1}=h_{\mathbf{i}^{\prime}} \circ f_{0} \circ g_{\mathbf{i}^{\prime}}^{-1}$ on $C_{\mathbf{i}^{\prime}}$, and therefore property (ii) in Subsection 3.5 and the definitions of $h_{\mathbf{i}}$ and $g_{\mathbf{i}}$ imply that in a neighbourhood of $\partial C_{\mathbf{i}}$

$$
f_{n-1}=h_{i_{n}} \circ h_{\mathbf{i}^{\prime}} \circ f_{0} \circ g_{\mathbf{i}^{\prime}}^{-1} \circ g_{i_{n}}^{-1}=h_{\mathbf{i}} \circ f_{0} \circ g_{\mathbf{i}}^{-1} \text {. }
$$

To prove statement (ii) it suffices to show that the maps $f_{n}$ form a Cauchy sequence with respect to the norm of $C^{1, \alpha}\left(\mathbb{R}^{d}\right)$ defined by (3.2) with $x_{0} \in \partial C_{0}$. Let us estimate the norm of $f_{n}-f_{n-1}$. By (3.8) this map vanishes outside the union of all $C_{\mathbf{i}}$ with $\mathbf{i} \in J^{n}$, and then

$$
\begin{aligned}
\left\|f_{n}-f_{n-1}\right\|_{C^{1, \alpha}\left(\mathbb{R}^{d}\right)} & =\sup _{\mathbf{i} \in J^{n}}\left\|\nabla f_{n}-\nabla f_{n-1}\right\|_{C_{\mathrm{hom}}^{0, \alpha}\left(C_{\mathbf{i}}\right)} \\
& \leq \sup _{\mathbf{i} \in J^{n}}\left[\left\|\nabla f_{n}\right\|_{C_{\mathrm{hom}}^{0, \alpha}\left(C_{\mathbf{i}}\right)}+\left\|\nabla f_{n-1}\right\|_{C_{\mathrm{hom}}^{0, \alpha}\left(C_{\mathbf{i}^{\prime}}\right)}\right] .
\end{aligned}
$$

By (3.8) the map $f_{n}$ agrees with $h_{\mathbf{i}} \circ f_{0} \circ g_{\mathbf{i}}^{-1}$ on each $C_{\mathbf{i}}$, and since $g_{\mathbf{i}}$ and $h_{\mathbf{i}}$ are homotheties with scaling factors $r^{n}$ and $\rho^{n}$, respectively ( $c f$. (3.7) and Subsection 3.4), 
using the interpolation inequality (3.3) we get

$$
\begin{aligned}
\left\|\nabla f_{n}\right\|_{C_{\text {hom }}^{0, \alpha}\left(C_{\mathbf{i}}\right)} & \leq 2\left\|\nabla f_{n}\right\|_{\infty}^{1-\alpha}\left\|\nabla^{2} f_{n}\right\|_{\infty}^{\alpha} \\
& =2\left(\frac{\rho^{n}}{r^{n}}\left\|\nabla f_{0}\right\|_{\infty}\right)^{1-\alpha}\left(\frac{\rho^{n}}{r^{2 n}}\left\|\nabla^{2} f_{0}\right\|_{\infty}\right)^{\alpha} \\
& =C\left(\frac{\rho}{r^{1+\alpha}}\right)^{n},
\end{aligned}
$$

where $C:=2\left\|\nabla f_{0}\right\|_{\infty}^{1-\alpha}\left\|\nabla^{2} f_{0}\right\|_{\infty}^{\alpha} \cdot{ }^{20}$

Putting together estimates (3.9) and (3.10) we get

$$
\left\|f_{n}-f_{n-1}\right\|_{C^{1, \alpha}\left(\mathbb{R}^{d}\right)} \leq 2 C\left(\frac{\rho}{r^{1+\alpha}}\right)^{n-1},
$$

and therefore assumption (3.6) implies that the sum of $\left\|f_{n}-f_{n-1}\right\|_{C^{1, \alpha}}$ over all $n=1,2, \ldots$ is finite, which implies that $\left(f_{n}\right)$ is a Cauchy sequence in $C^{1, \alpha}$.

We now prove statement (iii). Let $y \in Q_{0}$ be fixed. We choose a sequence of indexes $i_{n} \in J$ such that, for every $n$, the point $y$ belongs to $Q_{\mathbf{i}_{n}}$ with $\mathbf{i}_{n}:=$ $\left(i_{1}, \ldots, i_{n}\right)$, and we denote by $\bar{x}$ the only point in the intersection of the sets $C_{\mathbf{i}_{n}}$.

Let $n=0,1, \ldots$ be fixed. Since $f$ agrees with $h_{\mathbf{i}_{n}} \circ f_{0} \circ g_{\mathbf{i}_{n}}^{-1}$ on $\partial C_{\mathbf{i}_{n}}$, property (iii) in Subsection 3.5 implies that for every $j=1,2,3$ the set $f^{-1}(y) \cap g_{\mathbf{i}_{n}}\left(G_{j}\right)$ consists of only one point, denoted by $x_{j, n}$, and there exist pairwise disjoint curves parametrized by $\gamma_{j, n}$ joining $x_{j, n}$ to $x_{j, n+1}$ and contained in $E_{y} \cap \operatorname{Int}\left(C_{\mathbf{i}_{n}} \backslash C_{\mathbf{i}_{n+1}}\right)$ except for the end points.

Upon re-parametrization, we can assume that each $\gamma_{j, n}$ is defined on the interval $\left[2^{-n-1}, 2^{-n}\right]$ and satisfies $\gamma_{j, n}\left(2^{-n}\right)=x_{j, n}$ and $\gamma_{j, n}\left(2^{-n-1}\right)=x_{j, n+1}$. We then set

$$
\gamma_{j}(t):= \begin{cases}\gamma_{j, n}(t) & \text { for } 2^{-n-1}<t \leq 2^{-n}, n=0,1, \ldots \\ \bar{x} & \text { for } t=0 .\end{cases}
$$

It is easy to check that the paths $\gamma_{j}$ are continuous (the only issue being the continuity at $t=0$ ), and the associated curves are contained in the level set $E_{y}$ and have only the end point $\bar{x}$ in common. Hence the union of these curves is a triod contained in $E_{y}$.

A standard topological argument shows that a connected set in $\mathbb{R}^{d}$ that contains a triod cannot be a simple curve (and clearly not even a point).

\section{Examples of functions without the weak Sard property}

The critical set $S$ of a Lipschitz function $f: \mathbb{R}^{2} \rightarrow \mathbb{R}$ is the set of all $x \in \mathbb{R}^{2}$ where $f$ is not differentiable or $\nabla f(x)=0$. As explained in the introduction, we are

${ }^{20} C$ is finite because $f_{0}$ is a map of class $C^{2}$ on $\mathbb{R}^{d}$ with compact support. 
interested in the following property (that for functions of class $C^{2}$ is an immediate corollary of Sard's theorem): the push-forward according to $f$ of the restriction of $\mathscr{L}^{2}$ to $S$ is singular with respect to $\mathscr{L}^{1}$, that is

$$
f_{\#}\left(1_{S} \cdot \mathscr{L}^{2}\right) \perp \mathscr{L}^{1} .
$$

This property clearly implies the following weak Sard property [1, Section 2.13]:

$$
f_{\#}\left(1_{S \cap E^{*}} \cdot \mathscr{L}^{2}\right) \perp \mathscr{L}^{1},
$$

where the set $E^{*}$ is the union of all connected components with positive length of all level sets of $f$ (see Subsection 2.4).

As pointed out in [1, Section 2.15(iii) and Section 2.15(v)], property (4.1), and therefore also (4.2), hold whenever $f$ has the Lusin property with functions of class $C^{2}$, and in particular when $f$ is locally of class $W^{2,1}$.

In this section we show that in terms of Sobolev classes this regularity assumption is optimal: from Subsection 4.1 to 4.5 we construct an example of Lipschitz function $f$ without the weak Sard property (Proposition 4.7), and in Subsection 4.8 we show how to modify this construction so to obtain a function $f^{\prime}$ of class $C^{1, \alpha}$ for every $\alpha<1$, and therefore also of class $W^{\beta, p}$ for every $\beta<2$ and every $p \leq \infty$.

Finally, in Proposition 4.10 we prove that the class $\mathscr{F}$ of all Lipschitz functions $f: \mathbb{R}^{2} \rightarrow \mathbb{R}$ which satisfy the weak Sard property is residual in the Banach space of Lipschitz functions $\operatorname{Lip}\left(\mathbb{R}^{2}\right)$; this shows that in some sense $\operatorname{Lip}\left(\mathbb{R}^{2}\right)$ is the smallest Banach space of functions that contains $\mathscr{F}$ (Remark 4.11).

4.1. Construction parameters. For the rest of this section $\left(a_{n}\right),\left(b_{n}\right)$, and $\left(r_{n}\right)$ are decreasing sequences of positive real numbers such that

$$
a_{n} \sim b_{n} \sim r_{n} \sim \frac{1}{n^{2} 2^{n}},
$$

where $\sim$ stands for asymptotic equivalence. ${ }^{21}$ Hence the following sums are finite:

$$
\hat{a}:=\sum_{n=0}^{\infty} 2^{n+2} a_{n}, \quad \hat{b}:=\sum_{n=0}^{\infty} 2^{n+1} b_{n}, \quad \hat{r}:=\sum_{n=0}^{\infty} 2^{n+2} r_{n} .
$$

We also choose $\delta>0$, and set

$$
c_{0}:=\delta+\hat{a}+\hat{r}, \quad d_{0}:=\delta+\hat{b}+\hat{r} .
$$

4.2. Construction of the sets $C_{n}$ and $D_{n}$. The set $C_{0}$ is a closed rectangle with width $c_{0}$ and height $d_{0} ; D_{0}$ is the union of 4 closed rectangles (called the components of $D_{0}$ ) with width $\frac{1}{2} c_{0}-2 a_{0}$ and height $\frac{1}{2} d_{0}-b_{0}$ arranged as in Figure 4.1; $C_{1}$ is the union of 4 closed rectangles with width $c_{1}:=\frac{1}{2} c_{0}-2 a_{0}-2 r_{0}$ and height $d_{1}:=\frac{1}{2} d_{0}-b_{0}-2 r_{0}$, each one concentric to a component of $D_{0}$. And so on...

${ }^{21}$ For the purpose of constructing the function $f$ (see Subsection 4.5), the parameters $r_{n}$ could be taken equal to 0 ; the choice in (4.3) is relevant only in the construction of the function $f^{\prime}$ (see Subsection 4.8). 


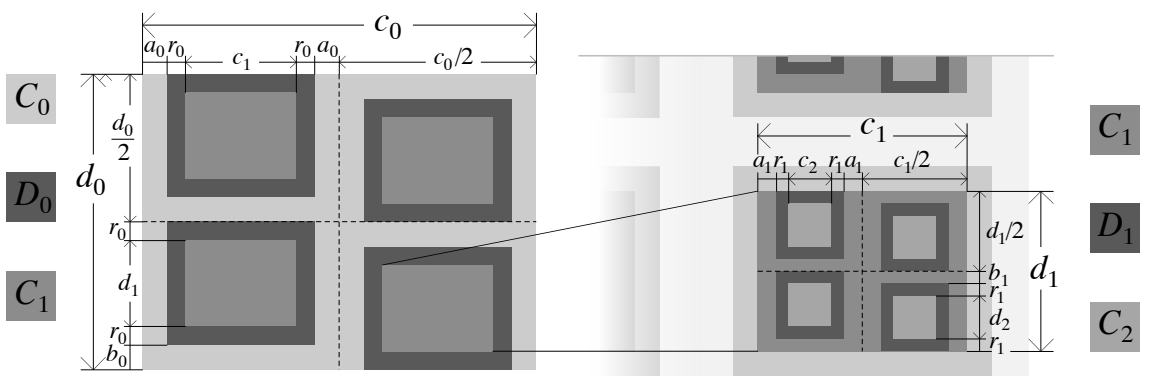

Figure 4.1. The sets $C_{i}$ for $i=0,1,2$, and $D_{i}$ for $i=0,1$.

Thus $C_{n}$ is the union of $4^{n}$ pairwise disjoint, closed rectangles with width $c_{n}$ and height $d_{n}$ (the components of $C_{n}$ ); $D_{n}$ is the union of $4^{n+1}$ pairwise disjoint, closed rectangles with width $\frac{1}{2} c_{n}-2 a_{n}$ and height $\frac{1}{2} d_{n}-b_{n}$, and each component of $C_{n}$ contains 4 components of $D_{n} ; C_{n+1}$ is the union of $4^{n+1}$ pairwise disjoint, closed rectangles with width $c_{n+1}$ and height $d_{n+1}$ given by

$$
c_{n+1}:=\frac{1}{2} c_{n}-2 a_{n}-2 r_{n}, \quad d_{n+1}:=\frac{1}{2} d_{n}-b_{n}-2 r_{n},
$$

and each of these rectangles is concentric to a component of $D_{n}$.

Taking into account the definition of $c_{0}$ and $d_{0}$ it follows immediately that

$$
2^{n} c_{n}=c_{0}-\sum_{m=0}^{n-1}\left(2^{m+2} a_{m}+2^{m+2} r_{m}\right) \searrow \delta,
$$

and

$$
2^{n} d_{n}=d_{0}-\sum_{m=0}^{n-1}\left(2^{m+1} b_{m}+2^{m+2} r_{m}\right) \searrow \delta .
$$

In particular $c_{n}$ and $d_{n}$ are always strictly positive, ${ }^{22}$ and satisfy

$$
c_{n} \sim d_{n} \sim \frac{\delta}{2^{n}}
$$

We denote by $C$ the intersection of the closed sets $C_{n}$. Thus (4.5) yields

$$
\mathscr{L}^{2}(C)=\lim _{n \rightarrow \infty} \mathscr{L}^{2}\left(C_{n}\right)=\lim _{n \rightarrow \infty} 4^{n} c_{n} d_{n}=\delta^{2} .
$$

4.3. Construction of the functions $f_{n}$ and $h_{n}$. We construct a sequence of Lipschitz and piecewise smooth functions $f_{n}: \mathbb{R}^{2} \rightarrow \mathbb{R}$ as follows. The function $f_{0}=f_{0}\left(x_{1}, x_{2}\right)$ agrees with $x_{2}$ in $\mathbb{R}^{2} \backslash C_{0}$, while in $C_{0}$ it is defined by its level curves, which are described in Figure 4.2(a); $f_{1}$ agrees with $f_{0}$ in $\mathbb{R}^{2} \backslash C_{1}$, while in each component of $C_{1}$ it is defined by its level curves, described in Figure 4.2(a). And so on for $n=2,3, \ldots$

22 This means that $C_{n}$ and $D_{n}$ are well-defined for every $n$. 
(a)

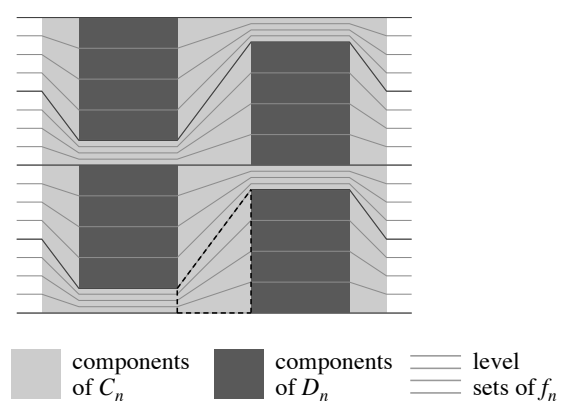

(b)

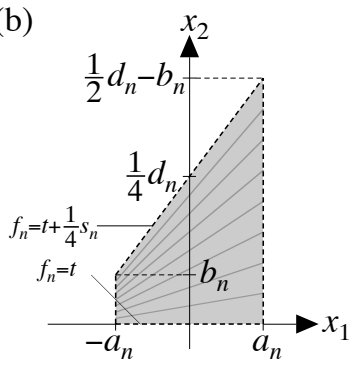

Figure 4.2. The level curves of $f_{n}$ in each connected component of $C_{n}$.

We denote by $s_{n}$ the oscillation of $f_{n}$ on the components of $C_{n} \cdot{ }^{23}$ On each component $R$ of $D_{n}$ the function $f_{n}$ is affine, depends only on the variable $x_{2}$, and is increasing in $x_{2}$. Hence $\nabla f_{n}=\left(0, v_{n}\right)$ for some $v_{n}>0$. Clearly the same holds for the components $R^{\prime}$ of $C_{n+1}$, and then

$$
v_{n}=\frac{\operatorname{osc}\left(f_{n}, R\right)}{\operatorname{height}(R)}=\frac{\operatorname{osc}\left(f_{n}, R^{\prime}\right)}{\operatorname{height}\left(R^{\prime}\right)} .
$$

Since height $(R)=d_{n+1}+2 r_{n}$, height $\left(R^{\prime}\right)=d_{n+1}, \operatorname{osc}\left(f_{n}, R\right)=\frac{1}{4} s_{n}$ (cf. Figure 4.2(a)), and $\operatorname{osc}\left(f_{n}, R^{\prime}\right)=s_{n+1}$, the second identity in the previous formula yields

$$
4 s_{n+1}=\frac{d_{n+1}}{d_{n+1}+2 r_{n}} s_{n},
$$

and taking into account that $s_{0}=d_{0}$ we get

$$
4^{n} s_{n}=d_{0}\left[\prod_{m=1}^{n} \frac{d_{m}}{d_{m}+2 r_{m-1}}\right] \searrow d_{0}[\underbrace{\prod_{m=1}^{\infty} \frac{d_{m}}{d_{m}+2 r_{m-1}}}_{\sigma}] .
$$

Using (4.3) and (4.5) we obtain $\log \left(d_{m} /\left(d_{m}+2 r_{m-1}\right)\right)=O\left(1 / m^{2}\right)$, and therefore the infinite product $\sigma$ is strictly positive. In particular, (4.7) implies

$$
s_{n} \sim d_{0} \sigma 4^{-n}
$$

Finally, for every $n=1,2, \ldots$ we set

$$
h_{n}:=f_{n}-f_{n-1} .
$$

23 This oscillation is clearly the same on all components of $C_{n}$. 
4.4. Estimate on the norm of $f_{n}$ and $h_{n}$. To begin with, we compute the $L^{\infty}$ norm of $\nabla f_{n}$ on the set $C_{n}$. Clearly it suffices to compute the supremum of $\left|\nabla f_{n}\right|$ on one component of $C_{n}$, and a closer inspection of Figure 4.2(a) shows that this supremum is attained in the set $E$ defined in the same figure. If we choose the axis as in Figure 4.2(b) and denote by $t$ the value of $f_{n}$ at the bottom of $E$, then we readily check that for every $x \in E, f_{n}(x)$ is given by

$$
f_{n}(x)=t+\frac{a_{n} s_{n} x_{2}}{a_{n} d_{n}+\left(d_{n}-4 b_{n}\right) x_{1}},
$$

and a straightforward computation yields

$$
\nabla f_{n}(x)=\frac{\left(-\left(d_{n}-4 b_{n}\right)\left(f_{n}(x)-t\right), a_{n} s_{n}\right)}{a_{n} d_{n}+\left(d_{n}-4 b_{n}\right) x_{1}} .
$$

Equations (4.3) and (4.5) imply $d_{n}-4 b_{n} \sim \delta 2^{-n}$; in particular $d_{n}-4 b_{n}>0$ for $n$ sufficiently large, and, taking into account that $x_{1} \geq-a_{n}$, the denominator in (4.10) is larger than $4 a_{n} b_{n}$. Since $\left|f_{n}-t\right| \leq s_{n}$, the absolute value of the first component of the numerator is smaller than $\left(d_{n}-4 b_{n}\right) s_{n}$. Hence (for $n$ sufficiently large)

$$
\left\|\nabla f_{n}\right\|_{L^{\infty}\left(C_{n}\right)} \leq \frac{s_{n}}{4 b_{n}}\left(\frac{d_{n}-4 b_{n}}{a_{n}}+1\right)=O\left(n^{4} 2^{-n}\right),
$$

where the final equality follows from equations (4.3) and (4.8).

Now we focus on the functions $h_{n}$ defined in (4.9). By construction, $f_{n}$ and $f_{n-1}$ agree outside $C_{n}$, and therefore the support of $h_{n}$ is contained in $C_{n}$. Then (4.11) yields

$$
\left\|\nabla h_{n}\right\|_{\infty} \leq\left\|\nabla f_{n}\right\|_{L^{\infty}\left(C_{n}\right)}+\left\|\nabla f_{n-1}\right\|_{L^{\infty}\left(C_{n-1}\right)}=O\left(n^{4} 2^{-n}\right),
$$

and since the distance of a point in $C_{n}$ from $\mathbb{R}^{2} \backslash C_{n}$ is of order $c_{n}=O\left(2^{-n}\right)$,

$$
\left\|h_{n}\right\|_{\infty}=O\left(n^{4} 4^{-n}\right) .
$$

4.5. Construction of $f$. We take the functions $f_{n}$ and $h_{n}$ as in Ssubsection 4.3, and for every $x \in \mathbb{R}^{2}$ we set

$$
f(x):=\lim _{n \rightarrow+\infty} f_{n}(x)=f_{0}(x)+\sum_{n=1}^{\infty} h_{n}(x) .
$$

Estimates (4.12) and (4.13) show that the sum of the norms $\left\|h_{n}\right\|_{C^{0,1}}$ (defined by (3.2) with an arbitrary choice of $x_{0}$ ) is finite and therefore $f$ is a well-defined Lipschitz function on $\mathbb{R}^{2}$.

Lemma 4.6. Let be given a compact metric space $X$ and a sequence of countable Borel partitions $\mathscr{F}_{n}$ of $X$. Let $\delta_{n}$ be the supremum of the diameters of the elements of $\mathscr{F}_{n}$, and assume that $\delta_{n} \rightarrow 0$ as $n \rightarrow+\infty$. Then two finite measures $\mu_{1}$ and $\mu_{2}$ on $X$ which agree on the elements of $\cup \mathscr{F}_{n}$ agree also on all Borel sets. 
Proof. For every $n$, let $\mathscr{G}_{n}$ be the class of all bounded functions on $X$ which are constant on each element of $\mathscr{F}_{n}$. Then $\int g d \mu_{1}=\int g d \mu_{2}$ for every $g \in \mathscr{G}_{n}$, and the equality carries over to all continuous functions $g$ because they can be uniformly approximated by functions in $\cup \mathscr{G}_{n}$.

Proposition 4.7. Take the set $C$ as in Subsection 4.2, and let $f$ be the function defined in (4.14). Then

(i) $f$ is differentiable at every $x \in C$ and $\nabla f(x)=0$;

(ii) $\mathscr{L}^{1}(f(C))=d_{0} \sigma$ where $\sigma$ is given in (4.7);

(iii) $f_{\#}\left(1_{C} \cdot \mathscr{L}^{2}\right)=m 1_{f(C)} \cdot \mathscr{L}^{1}$ where $m:=\delta^{2} /\left(d_{0} \sigma\right)$; in particular, $f$ does not satisfy the weak Sard property (4.2).

Proof. (i) For every $n \geq 0$, we write $f$ as $f_{n}+h_{n+1}+h_{n+2}+\cdots$, and then estimates (4.11) and (4.12) yield

$$
\|\nabla f\|_{L^{\infty}\left(C_{n}\right)}=O\left(n^{4} 2^{-n}\right) .
$$

This implies that the Lipschitz constant of $f$ on each component of $C_{n}$ is of order $O\left(n^{4} 2^{-n}\right)$; since $C$ is contained in the interior of $C_{n}$, it follows that

$$
\limsup _{y \rightarrow x} \frac{|f(y)-f(x)|}{|x-y|}=O\left(n^{4} 2^{-n}\right)
$$

for every $x \in C$, and taking the limit as $n \rightarrow+\infty$ we obtain that $f$ is differentiable at $x$ and $\nabla f(x)=0$.

(ii) We note that $f(C)$ is the intersection of all $f\left(C_{n}\right)$, and $f\left(C_{n}\right)$ agrees with $f_{n}\left(C_{n}\right)$, and therefore it is the union of $4^{n}$ pairwise disjoint, closed interval with length $s_{n}$ - the images according to $f$ of the components of $C_{n}$. Therefore, using (4.8) we get

$$
\mathscr{L}^{1}(f(C))=\lim _{n \rightarrow+\infty} \mathscr{L}^{1}\left(f_{n}\left(C_{n}\right)\right)=\lim _{n \rightarrow+\infty} 4^{n} s_{n}=d_{0} \sigma .
$$

(iii) We must show that the measures $\mu:=f_{\#}\left(1_{C} \cdot \mathscr{L}^{2}\right)$ and $\lambda:=m 1_{f(C)} \cdot \mathscr{L}^{1}$ are the same. Since both $\mu$ and $\lambda$ are supported on the compact set $f(C)$, we apply Lemma 4.6 to the partitions $\mathscr{F}_{n}$ given by the sets $R^{\prime}:=f(R \cap C)$ where $R$ is a component of $C_{n}$, and deduce that it suffices to prove $\mu\left(R^{\prime}\right)=\lambda\left(R^{\prime}\right)$ for every such $R^{\prime}$.

Since $C$ can be written as a disjoint union of $4^{n}$ translated copies of $R \cap C$, we have

$$
\mu\left(R^{\prime}\right)=\mathscr{L}^{2}(R \cap C)=4^{-n} \mathscr{L}^{2}(C)=4^{-n} \delta^{2} .
$$

On the other hand $f(C)$ can be written as a disjoint union of $4^{n}$ translated copies of $R^{\prime}$, and then

$$
\lambda\left(R^{\prime}\right)=m \mathscr{L}^{1}\left(R^{\prime}\right)=4^{-n} m \mathscr{L}^{1}(f(C))=4^{-n} m d_{0} \sigma=4^{-n} \delta^{2} .
$$


In the next subsections we show how to modify the construction in Subsection 4.5 so to obtain a more regular example.

4.8. Construction of $f^{\prime}$. For every $n=0,1,2, \ldots$ we consider the smoothing kernels

$$
\rho_{n}(x):=r_{n}^{-2} \rho\left(x / r_{n}\right),
$$

where $r_{n}$ is given in Subsection 4.1 and $\rho: \mathbb{R}^{2} \rightarrow \mathbb{R}$ is a positive smooth function which satisfies the following conditions:

(a) the support of $\rho$ is contained in the ball with center 0 and radius $1 / 2$;

(b) $\int_{\mathbb{R}^{2}} \rho(x) d x=1$ and $\int_{\mathbb{R}^{2}} x \rho(x) d x=0$.

Then we take $f_{n}$ and $h_{n}$ as in Subsection 4.3 , and define $f^{\prime}: \mathbb{R}^{2} \rightarrow \mathbb{R}$ as

$$
f^{\prime}:=f_{0} * \rho_{0}+\sum_{n=1}^{\infty} h_{n} * \rho_{n},
$$

where $*$ denotes the usual convolution product.

Proposition 4.9. Take $C$ and $f$ as in Subsection 4.2 and Subsection 4.5, and let $f^{\prime}$ be the function defined by (4.15). Then

(i) $f^{\prime}$ is a well-defined function of class $C^{1, \alpha}$ for every $\alpha<1$;

(ii) $f^{\prime}$ is smooth on $\mathbb{R}^{2} \backslash C$ and agrees with $f$ on $C$;

(iii) $\nabla f^{\prime}(x)=0$ for every $x \in C$;

(iv) $f_{\#}^{\prime}\left(1_{C} \cdot \mathscr{L}^{2}\right)=m 1_{f^{\prime}(C)} \cdot \mathscr{L}^{1}$ where $m:=\delta^{2} /\left(d_{0} \sigma\right)$; in particular, $f^{\prime}$ does not satisfy the weak Sard property (4.2).

Proof. (i) We claim that the series $\sum h_{n} * \rho_{n}$ converge in norm in the Banach space $C^{1, \alpha}$ for every $\alpha<1$, and therefore $f^{\prime}$ belongs to this space. Indeed, using (3.3), (4.12), and (4.3) we obtain

$$
\begin{aligned}
\left\|\nabla\left(h_{n} * \rho_{n}\right)\right\|_{C_{\mathrm{hom}}^{0, \alpha}} & \leq 2\left\|\nabla\left(h_{n} * \rho_{n}\right)\right\|_{\infty}^{1-\alpha}\left\|\nabla^{2}\left(h_{n} * \rho_{n}\right)\right\|_{\infty}^{\alpha} \\
& \leq 2\left(\left\|\nabla h_{n}\right\|_{\infty}\left\|\rho_{n}\right\|_{1}\right)^{1-\alpha}\left(\left\|\nabla h_{n}\right\|_{\infty}\left\|\nabla \rho_{n}\right\|_{1}\right)^{\alpha} \\
& =2\left\|\nabla h_{n}\right\|_{\infty}\left\|\nabla \rho_{n}\right\|_{1}^{\alpha} \\
& =\left\|\nabla h_{n}\right\|_{\infty} O\left(r_{n}^{-\alpha}\right)=O\left(n^{4+2 \alpha} 2^{-(1-\alpha) n}\right) .
\end{aligned}
$$

Moreover estimates (4.12) and (4.13) hold even if $h_{n}$ is replaced by $h_{n} * \rho_{n}$, and therefore $\left\|h_{n} * \rho_{n}\right\|_{C^{1, \alpha}}=O\left(n^{6} 2^{-(1-\alpha) n}\right)$, which implies that the sum of the norms $\left\|h_{n} * \rho_{n}\right\|_{C^{1, \alpha}}$ is finite.

(ii) Each $h_{n}$ is supported in $C_{n}$ (cf. Subsection 4.3) and since $\rho_{n}$ is supported in the ball with center 0 and radius $r_{n} / 2$, the function $h_{n} * \rho_{n}$ is supported in the closed set $C_{n}^{\prime}$ of all points whose distance from $C_{n}$ is at most $r_{n} / 2$. Since the sets $C_{n}^{\prime}$ decrease 
to $C, f^{\prime}$ agrees with a finite sum of smooth functions in every open set with positive distance from $C$, and therefore is smooth on $\mathbb{R}^{2} \backslash C$.

By construction, $f_{n}$ is affine on $D_{n}$ and then $h_{n}=f_{n}-f_{n-1}$ is affine on $D_{n}$ too. Therefore, assumption (b) in Subsection 4.8 and the fact that $\rho_{n}$ is supported in the ball with center 0 and radius $r_{n} / 2$ imply that $h_{n} * \rho_{n}=h_{n}$ in the closed set $D_{n}^{\prime}$ of all points of $D_{n}$ whose distance from the boundary of $D_{n}$ is at least $r_{n} / 2$. Since $D_{n}^{\prime}$ contains $C_{n+1}\left(c f\right.$. Figure 4.1), which in turn contains $C$, we have that $h_{n} * \rho_{n}=h_{n}$ on $C$. A similar argument shows that $f_{0} * \rho_{0}=f_{0}$ on $C$. Then $f=f^{\prime}$ on $C$ by definitions (4.14) and (4.15).

Since $f^{\prime}=f$ on $C$, statements (iii) and (iv) follow (almost) immediately from statements (i) and (iii) of Proposition 4.7.

The next result that shows that the weak Sard property is verified by a generic function.

Proposition 4.10. Let $\operatorname{Lip}\left(\mathbb{R}^{2}\right)$ be the Banach space of Lipschitz function on $\mathbb{R}^{2}$, and let $\mathscr{G}$ be the subclass of all functions whose critical set has measure zero. Then $\mathscr{G}$ is residual in $\operatorname{Lip}\left(\mathbb{R}^{2}\right){ }^{24}$

Proof. For every $r>0$ let $\mathscr{G}_{r}$ be the class of all $f \in \operatorname{Lip}\left(\mathbb{R}^{2}\right)$ whose critical set $S(f)$ satisfies $\mathscr{L}^{2}(S(f))<r$. Since $\mathscr{G}$ is the intersection of all $\mathscr{G}_{r}$ with $r=1 / n$ and $n=1,2, \ldots$, it suffices to show that each $\mathscr{G}_{r}$ is open and dense in $\operatorname{Lip}\left(\mathbb{R}^{2}\right)$. Among the many (equivalent) complete norms on $\operatorname{Lip}\left(\mathbb{R}^{2}\right)$ we consider the following: $\|f\|:=|f(0)|+\|\nabla f\|_{\infty}$.

Step 1: $\mathscr{G}_{r}$ is open. Take $f \in \mathscr{G}_{r}$. Since $\mathscr{L}^{2}(S(f))<r$, there exists $\delta>0$ such that $\mathscr{L}^{2}(\{x:|\nabla f(x)| \leq \delta\})<r$, and therefore $\mathscr{L}^{2}(S(g))<r$ for every $g \in \operatorname{Lip}\left(\mathbb{R}^{2}\right)$ such that $\|\nabla f-\nabla g\|_{\infty}<\delta$, that is, for every $g$ in a neighbourhood of $f$.

Step 2: $\mathscr{G}_{r}$ is dense. Given $f \in \operatorname{Lip}\left(\mathbb{R}^{2}\right)$ and $\delta>0$, we must find $g \in \mathscr{G}_{r}$ such that $\|g-f\| \leq \delta$. We take an open set $A$ which contains $S(f)$ and satisfies $\mathscr{L}^{2}(A \backslash$ $S(f))<r$, and choose a sequence of pairwise disjoint closed discs $D_{n}$ contained in $A$ which cover almost all of $S(f)$. Then for every $n$ we choose a Lipschitz function $g_{n}$ such that $\nabla g_{n} \neq 0$ a.e. in $D_{n}$ and $\nabla g_{n}=0$ a.e. in $\mathbb{R}^{2} \backslash D_{n}$. Up to rescaling we can assume that $\left\|g_{n}\right\| \leq 2^{-n} \delta$, and then we set

$$
g:=f+\sum_{n=1}^{\infty} g_{n} .
$$

Thus $g$ is Lipschitz, $\|g-f\| \leq \delta$, and $S(g)$ is contained in $A \backslash S(f)$, and in particular $\mathscr{L}^{2}(S(g))<r$, that is, $g$ belongs to $\mathscr{G}_{r}$.

24 A set in a topological space is residual if it contains a countable intersection of open dense sets. By Baire theorem, a residual set in a complete metric space is dense. 
Remark 4.11. (i) The class $\mathscr{F}$ of all functions in $\operatorname{Lip}\left(\mathbb{R}^{2}\right)$ which satisfy the weak Sard property contains $\mathscr{G}$, and therefore it is residual in $\operatorname{Lip}\left(\mathbb{R}^{2}\right)$.

(ii) $\operatorname{Lip}\left(\mathbb{R}^{2}\right)$ is the smallest functional space which contains $\mathscr{F}$. More precisely, a Banach space $X$ which embeds continuously in $\operatorname{Lip}\left(\mathbb{R}^{2}\right)$ and (whose image) contains $\mathscr{F}$ must agree with $\operatorname{Lip}\left(\mathbb{R}^{2}\right)$ itself. Indeed the image of $X$ is residual in $\operatorname{Lip}\left(\mathbb{R}^{2}\right)$, the Open Mapping Theorem implies that the embedding is open, and therefore an isomorphism.

\section{Non-locality of the divergence operator}

In this section we show that the divergence operator is not strongly local on the space of vector fields in $L^{\infty}$ with distributional divergence in $L^{\infty}$. More precisely, in Subsection 5.4 we construct a bounded vector field $b$ on the plane whose distributional divergence $\operatorname{div} b$ is bounded, non-trivial, and its support is contained in the set where $b$ takes the value 0 (Proposition 5.6).

Remark 5.1. Proposition 6.6 in [1] establishes the following relation between the locality of the divergence and the weak Sard property: let $b$ be a bounded vector field on the plane which has bounded divergence and can be represented as $b=$ $a \nabla^{\perp} f$ with $a$ a bounded function and $f$ a Lipschitz function; if $f$ satisfies the weak Sard property then the divergence of $b$ is local, in the sense that $\operatorname{div} b=0$ a.e. on every set where $b$ is a.e. constant.

Note that the vector field $b$ given in Subsection 5.4 can be written as $b=$ $1_{E} \nabla^{\perp} f$ where the set $E$ and the Lipschitz function $f$ are given in Subsection 5.3 and Subsection 5.7, respectively, and indeed $f$ does not satisfy the weak Sard property (in Proposition 5.9 we give a direct proof of this fact).

5.2. Construction parameters. For the rest of this section $\left(d_{n}\right)$ is a fixed, decreasing sequence of positive real numbers such that

$$
d_{n} \geq 5 \cdot 2^{-n} \text { for } n=0,1,2, \ldots
$$

and the sums

$$
c_{0}:=\sum_{n=0}^{\infty} 2^{n} d_{2 n}, \quad c_{1}:=\sum_{n=0}^{\infty} 2^{n} d_{2 n+1}
$$

are finite. We then choose $r>0$, and set $a_{0}:=c_{0}+\sqrt{2} r, a_{1}:=c_{1}+r$.

5.3. Construction of the sets $C$ and $E$. The set $C_{0}$ is a closed rectangle with width $a_{1}$ and height $a_{0} ; C_{1}$ is the union of two closed rectangles obtained by removing from $C_{0}$ the middle rectangle with same width (that is, $a_{1}$ ) and height $d_{0} ; C_{2}$ is the union of four closed rectangles obtained by removing from each of the two rectangles that compose $C_{1}$ the middle rectangle with same height (denoted by $a_{2}$ ) and width $d_{1}$ as shown in Figure 5.1. And so on... 
(a)

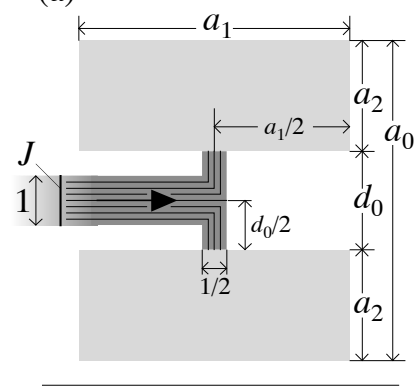

$C_{1} \quad E_{1} \quad \begin{aligned} & \text { integral } \\ & \text { curves of } b_{1}\end{aligned}$ (b)

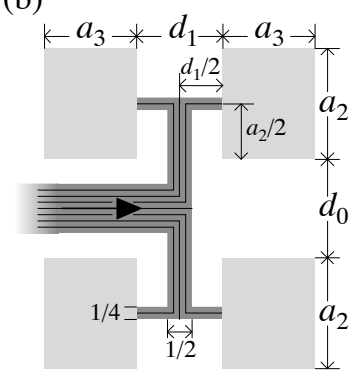

$C_{2} \quad E_{2} \quad \begin{aligned} & \text { integral } \\ & \text { curves of } b_{2}\end{aligned}$ (c)

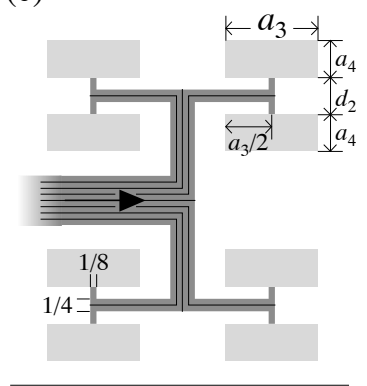

$C_{3} \quad E_{3} \quad \begin{aligned} & \text { integral } \\ & \text { curves of } b_{3}\end{aligned}$

Figure 5.1. The sets $C_{i}, E_{i}$, and the integral curves of $b_{i}$ (within $E_{i}$ ) for $i=1,2,3$.

Thus $C_{n}$ is contained in $C_{n-1}$, and consists of $2^{n}$ closed rectangles with the same width and the same height, called components of $C_{n}$. Let $a_{n}$ denote the width of such rectangles for $n$ odd, and the height for $n$ even (thus the other dimension is $\left.a_{n+1}\right)$; then $a_{n}$ satisfies the recursive relation

$$
2 a_{n+2}=a_{n}-d_{n}
$$

We easily deduce from (5.3) that for every $n \geq 1$

$$
2^{n} a_{2 n}=a_{0}-\sum_{m=0}^{n-1} 2^{m} d_{2 m} \searrow \sqrt{2} r
$$

and

$$
2^{n} a_{2 n+1}=a_{1}-\sum_{m=0}^{n-1} 2^{m} d_{2 m+1} \searrow r .
$$

Hence $a_{n}$ is strictly positive and

$$
a_{n} \sim 2^{(1-n) / 2} r
$$

The set $E_{n}$ is defined as in Figure 5.1, it is open, contained in the complement of $C_{n}$, and contains $E_{n-1}$. Note that $E_{1}-$ and therefore all $E_{n}$ - contains an horizontal strip unbounded to the left.

Finally, we denote by $C$ the intersection of the closed sets $C_{n}$, and by $E$ the union of the open s ets $E_{n}$. Thus $C$ is the product of two Cantor-like sets, and (5.4) yields

$$
\mathscr{L}^{2}(C)=\lim _{n \rightarrow \infty} \mathscr{L}^{2}\left(C_{n}\right)=\lim _{n \rightarrow \infty} 2^{n} a_{n} a_{n+1}=\sqrt{2} r^{2}
$$


5.4. Construction of the vector field $b$. For every $n \geq 0$, the vector field $b_{n}$ is set equal to 0 in $\mathbb{R}^{2} \backslash E_{n}$, while in $E_{n}$ it is piecewise constant, satisfies $\left|b_{n}\right|=1$ everywhere, and its direction can be inferred from the integral curves and the arrows drawn in Figure 5.1..$^{25}$ Note that $b_{n}$ agrees with $b_{n-1}$ in $E_{n-1}$ for every $n$.

Finally, the vector field $b$ is the pointwise limit of $b_{n} \cdot{ }^{26}$

The result in the next subsection is used to compute the divergence of $b .^{27}$

5.5. Divergence of piecewise regular vector fields. Let $b$ be a bounded vector field on $\mathbb{R}^{d}$, and $\left\{A_{i}\right\}$ a finite family of pairwise disjoint open sets with Lipschitz boundaries whose closures cover $\mathbb{R}^{d}$. We assume that $b$ agrees on each $A_{i}$ with a vector field $b_{i}$ of class $C^{1}$ defined on $\mathbb{R}^{d}$. Then the divergence of $b$ is a measure of the form

$$
\operatorname{div} b=h \cdot \mathscr{L}^{d}+g 1_{\Sigma} \cdot \mathscr{H}^{d-1},
$$

where $\Sigma$ is the union of all $\partial A_{i}, h$ is the pointwise divergence of $b$ (defined for all $x \notin \Sigma$ ), and $g$ is the difference of the normal components of $b$ at the two sides of $\Sigma$. More precisely, for every $x \in \partial A_{i} \cap \partial A_{j}$ where both $\partial A_{i}$ and $\partial A_{j}$ admit a tangent plane $T$, we set $g(x):=\left(b_{i}(x)-b_{j}(x)\right) \cdot \eta$ where $\eta$ is the unit normal to $T$ that points toward $A_{i}$.

Proposition 5.6. Take $C$ and $b$ as in Subsection 5.3 and Subsection 5.4. Then $b=0$ on $C$, while div $b$ is non trivial, belongs to $L^{\infty}\left(\mathbb{R}^{2}\right)$, and is supported on $C$. More precisely div $b=m 1_{C}$ where $m:=1 /\left(\sqrt{2} r^{2}\right)$.

Proof. The fact that $b=0$ on $C$ follows immediately from the fact that $b_{n}=0$ on $C$ by construction and $b$ is the pointwise limit of $b_{n}$.

Step 1: computation of the divergence of $b_{n}$. We compute div $b_{n}$ using formula (5.6). In this case $h=0$ because $b_{n}$ is piecewise constant, and $\Sigma$ consists of the boundary of $E_{n}$ plus the discontinuity points of $b_{n}$ contained in $E_{n}$, that is, the corner points of the integral curves drawn in Figure 5.1. Moreover $g=1$ in $F_{n}:=\partial E_{n} \cap \partial C_{n}$, and $g=0$ everywhere else. Thus $\operatorname{div} b_{n}=\mu_{n}$, where $\mu_{n}$ is the restriction of $\mathscr{H}^{1}$ to the set $F_{n}$. Since $F_{n}$ is the union of $2^{n}$ segments of length $2^{-n}, \mu_{n}$ is a probability measure.

Step 2: computation of the divergence of $b$. Since $b_{n}$ converge to $b$ in $L^{1}$, the divergence of $b$ is the limit (in the sense of distributions) of $\operatorname{div} b_{n}=\mu_{n}$ and therefore is a probability measure, which we denote by $\mu$. Since the support of each $\mu_{n}$ is contained in $C_{n}$, the support of $\mu$ is contained in $C$.

Take $n=1,2, \ldots$, and let $R$ be one of the $2^{n}$ components of $C_{n}$. We claim that

$$
\mu(R \cap C)=\mu(R)=2^{-n} .
$$

25 In what follows it does not matter how $b_{n}$ is defined at the corners of the integral curves.

${ }^{26}$ By construction the sequence $b_{n}(x)$ is definitely constant for every $x \in \mathbb{R}^{2}$, and therefore it converges to some limit $b(x)$. It can be easily shown that it is a Cauchy sequence in $L^{1}\left(\mathbb{R}^{2}\right)$.

27 Through this section, divergence, gradient, and curl are intended in the sense of distributions. 
Indeed, for every $m \geq n$, the closed rectangle $R$ contains $2^{m-n}$ of the $2^{m}$ segments that compose $F_{m}$. Hence $\mu_{m}(R)=2^{-n}$, and passing to the limit as $m \rightarrow+\infty$ we obtain $\mu(R) \geq 2^{-n}$. On the other hand, if $R^{\prime}$ is an open neighbourhood of $R$ which intersect no other component of $C_{n}$ we have $\mu_{m}\left(R^{\prime}\right)=2^{-n}$ for every $m \geq n$, and passing to the limit we get $\mu(R) \leq \mu\left(R^{\prime}\right) \leq 2^{-n}$. Thus (5.7) is proved.

Now we must show that $\mu=\lambda$ where $\lambda:=m 1_{C} \cdot \mathscr{L}^{2}$. Since $\mu$ and $\lambda$ are supported on $C$, by Lemma 4.6 it suffices to show that $\mu(R \cap C)=\lambda(R \cap C)$ for every rectangle $R$ taken as in Step 2. Since $C$ can be written as disjoint union of $2^{n}$ translated copies of $R \cap C$, then

$$
\lambda(R \cap C)=m \mathscr{L}^{2}(R \cap C)=2^{-n} m \mathscr{L}^{2}(C)=2^{-n}=\mu(R \cap C),
$$

where the third identity follows from (5.5), and the fourth one from (5.7).

5.7. Construction of the function $f$. By Proposition 5.6, the divergence of $b$ vanishes on the open set $E$, and therefore the rotated vector field $b^{\top}$ (see footnote 3 ) is curl-free on $E$. Since the set $E$ is simply connected, $b^{\top}$ is the gradient of a function $f: E \rightarrow \mathbb{R}$ of class $W^{1, \infty}$, and therefore we can assume that $f$ is locally Lipschitz on $E$.

Clearly $b=\nabla^{\perp} f$ a.e. on $E$, and the level sets of $f$ agree (in $E$ ) with the integral curves of $b$.

Since $f$ has Lipschitz constant 1 on $E$ (see Lemma 5.8 below), it can be extended to a function on the plane with Lipschitz constant 1 using McShane lemma.

Lemma 5.8. Take $f$ and $E$ as above. Then $f$ has Lipschitz constant 1 on E.

Proof. Since $|\nabla f|=|b|=1$ a.e. on $E$, the restriction of $f$ to every convex subset of $E$ has Lipschitz constant 1, but proving that $f$ has Lipschitz constant 1 on $E$ is more delicate.

Given $t_{i} \in f(E)$ for $i=1,2$, let $L_{i}:=\left\{x \in E: f(x)=t_{i}\right\}$ be the corresponding level sets, and set $\delta:=\left|t_{1}-t_{2}\right|$. Then $f$ has Lipschitz constant 1 if for every choice of $t_{i}$ and of $x_{i} \in L_{i}$ there holds

$$
\left|x_{1}-x_{2}\right| \geq \delta
$$

By a density argument, it suffices to prove (5.8) when both level sets intersect $C_{n}$ for all $n$.

Let $n$ be the smallest integer such that $L_{1} \cap C_{n}$ and $L_{2} \cap C_{n}$ are contained in different components of $C_{n}$. Denote these components by $R_{1}$ and $R_{2}$, respectively, and take the rectangle $R$ as in Figure 5.2(a). This picture clearly shows that if both $x_{1}$ and $x_{2}$ belong to $R_{1} \cup R_{2} \cup R$ then (5.8) holds.

We assume now that $x_{1} \notin R_{1} \cup R_{2} \cup R$ (the case $x_{2} \notin R_{1} \cup R_{2} \cup R$ is equivalent). Take $m$ such that $x_{1}$ belongs to the rectangle $S$ in Figure 5.2(b). There are two possibilities: either $x_{2}$ belongs to the rectangle $S^{\prime}$ defined in Figure 5.2(b), or it 
(a)

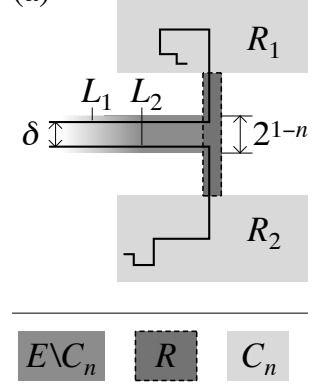

(b)

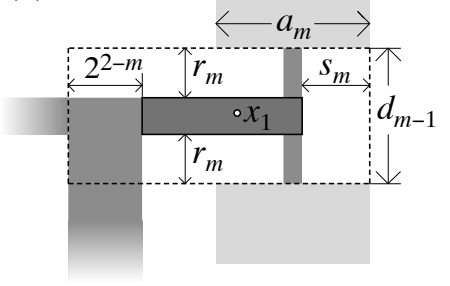

$\begin{array}{lll:l:l}E \backslash C_{m} & S & S^{\prime} & C_{m}\end{array}$ (c)

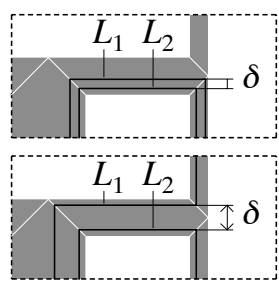

$S^{\prime}$

Figure 5.2. Estimating the distance between the level sets $L_{1}$ and $L_{2}$.

doesn't. In the former case, Figure 5.2(c) shows that (5.8) holds. ${ }^{28}$ In the latter case, Figure 5.2(b) shows that (5.8) holds provided that the lengths $r_{m}, s_{m}$ (defined in the figure) and $2^{2-m}$ are larger than $\delta$.

Let us prove these inequalities. Since $m \leq n$ and $2^{1-n} \geq \delta$ (see Figure 5.2(a)), then

$$
2^{2-m} \geq 2^{2-n} \geq 2 \delta
$$

Moreover assumption (5.1) yields

$$
r_{m}=\frac{1}{2}\left(d_{m-1}-2^{1-m}\right) \geq 2^{2-m} \geq 2 \delta .
$$

Finally, (5.3) and the positivity of $a_{n}$ imply $a_{n} \geq d_{n}$, and taking into account (5.1)

$$
s_{m}=\frac{1}{2}\left(a_{m}-2^{-m}\right) \geq \frac{1}{2}\left(d_{m}-2^{-m}\right) \geq 2^{1-m} \geq \delta .
$$

Proposition 5.9. Take $C, E, b$ and $f$ as in the previous subsections. Then

(i) $b=1_{E} \nabla^{\perp} f$ a.e.;

(ii) $\nabla f=0$ a.e. in $C$;

(iii) $f$ does not satisfy the weak Sard property.

Proof. Statement (i) follows immediately from the definitions of $b$ and $f$.

(ii) Let $\left\{e_{1}, e_{2}\right\}$ be the standard basis of $\mathbb{R}^{2}$. Fix a point $x \in C$ where $f$ is differentiable, and for every $n=1,2, \ldots$ denote by $R_{n}$ the connected component of $C_{n}$ which contains $x$.

Note that $R_{n}$ has sides of length $a_{n}$ and $a_{n+1}$ and since $a_{n} \geq a_{n+1}$, at least one of the points $x \pm \frac{1}{2} a_{n+1} e_{1}$ belongs to $R_{n}$. We denote this point by $x_{n}$. Then (5.4) yields

$$
\left|f\left(x_{n}\right)-f(x)\right| \sim\left|D_{1} f(x)\right| \frac{a_{n+1}}{2} \sim\left|D_{1} f(x)\right| \frac{r}{2} 2^{-n / 2} .
$$

28 The upper picture in Figure 5.2(c) refers to the case $m<n$ and the lower one to the case $m=n$. 
On the other hand, Figure 5.1 shows that the integral curves of $b$ (in $E$ ) which intersect $R_{n}$ are exactly those which intersect a certain (open) segment $J_{n}$ of length $2^{-n}$ contained in the segment $J$ in Figure 5.1(a). Taking into account that these integral curves are the restriction to $E$ of the level sets of $f$, we deduce that $f(C \cap$ $\left.R_{n}\right)=f\left(J_{n}\right)$, and since the slope of $f$ along $J_{n}$ is 1 , the set $f\left(J_{n}\right)$ is an interval of length $2^{-n}$, that is

$$
\operatorname{osc}\left(f, C \cap R_{n}\right)=\operatorname{osc}\left(f, J_{n}\right)=2^{-n},
$$

where $\operatorname{osc}(f, E)$ stands for the oscillation of $f$ on the set $E$.

A close examination of Figure 5.1 shows that the distance of any point of $R_{n}$ from $C \cap R_{n}$ is at most $\frac{1}{2}\left(d_{n}+d_{n+1}\right)$. Moreover $d_{n}=o\left(2^{-n / 2}\right)$ because we assumed that the sums in (5.2) converge. Therefore (5.10) and the fact that the Lipschitz constant of $f$ is 1 yields

$$
\operatorname{osc}\left(f, R_{n}\right) \leq \operatorname{osc}\left(f, C \cap R_{n}\right)+d_{n}+d_{n+1}=o\left(2^{-n / 2}\right) .
$$

Now, (5.9) and (5.11) can be both true only if $D_{1} f(x)=0$. A similar argument shows that $D_{2} f(x)=0$. We conclude that $\nabla f(x)=0$ for every $x \in C$ where $f$ is differentiable, that is, for a.e. $x \in C$.

(iii) As pointed out before, $f(C)=f(J)$ is an interval of length 1 , and therefore, upon addition of a constant, we can assume that this interval is $[0,1]$. To prove statement (iii) we show that $\mu:=f_{\#}\left(1_{C} \cdot \mathscr{L}^{2}\right)$ agrees with $m 1_{[0,1]} \cdot \mathscr{L}^{1}$ where $m:=\mathscr{L}^{2}(C)$.

To this end, it suffices to show that $\mu$ is non atomic and $\mu\left(I_{n, k}\right)=m / 2^{n}$ for every interval $I_{n, k}:=\left((k-1) 2^{-n}, k 2^{-n}\right)$ with $n=1,2, \ldots$ and $k=1, \ldots, 2^{n}$ (cf. Lemma 4.6).

The latter claim follows by the fact that $C \cap f^{-1}\left(I_{n, k}\right)$ agrees up to a null set with $C \cap R_{n, k}$ where $R_{n, k}$ is a suitable connected component of $C_{n}$, and therefore $\mathscr{L}^{2}\left(C \cap R_{n, k}\right)=m 2^{-n}$. The former claim follows by a similar argument.

\section{Appendix: a measurability lemma}

Let be given a compact metric space $X$, a topological space $Y$, a continuous map $f: X \rightarrow Y$, and a real number $d \geq 0$. For every $y \in Y$ we write $E_{y}$ for the level set $f^{-1}(y)$, and $E_{y}^{d}$ for union of all connected components $C$ of $E_{y}$ such that $\mathscr{H}^{d}(C)>0$, and then we let $E^{d}$ be the union of $E_{y}^{d}$ over all $y \in Y$.

Proposition 6.1. The sets $E_{y}^{d}$ and $E^{d}$ are countable unions of closed sets in $X$; in particular they are Borel measurable.

In order to prove this statement we need to recall some definitions and known facts. As usual $\mathscr{F}(X)$ is the class of all non-empty closed subsets of $X$, and is endowed with the Hausdorff distance $d_{H}$ (see (2.1)). Since $X$ is compact, the metric 
space $\mathscr{F}(X)$ is compact, and the subclass $\mathscr{F}_{c}(X)$ of all elements of $\mathscr{F}(X)$ which are connected is closed (see for instance [8, Theorems 3.16 and 3.18]).

Lemma 6.2. If $\mathscr{G}$ is a closed subclass of $\mathscr{F}(X)$, then the union $C$ of all sets in $\mathscr{G}$ is a closed subset of $X$.

Proof. Let $x$ be the limit of a sequence of points $x_{n} \in C$. For every $n$ there exists $C_{n} \in \mathscr{G}$ such that $x_{n} \in C_{n}$, and since $\mathscr{G}$ is closed in $\mathscr{F}(X)$ and therefore compact, the sequence $\left(C_{n}\right)$ has an accumulation point $C_{\infty} \in \mathscr{G}$. Hence $x$ belongs to $C_{\infty}$, and therefore also to $C$.

For every set $E$ in $X$ the $d$-dimensional Hausdorff measure $\mathscr{H}^{d}(E)$ is defined (up to a renormalization factor) as the supremum over all $\delta>0$ of the outer measures $\mathscr{H}_{\delta}^{d}(E)$ given by

$$
\mathscr{H}_{\delta}^{d}(E):=\inf \left\{\sum_{i}\left(\operatorname{diam}\left(A_{i}\right)\right)^{d}\right\}
$$

where the infimum is taken over all countable coverings $\left\{A_{i}\right\}$ of $E$ such that $\operatorname{diam}\left(A_{i}\right) \leq \delta$ for every $i$. Note that the value of $\mathscr{H}_{\delta}^{d}(E)$ does not change if the sets $A_{i}$ are assumed to be open.

Lemma 6.3. The outer measures $\mathscr{H}_{\delta}^{d}$ are upper semicontinuous on $\mathscr{F}(X)$.

Proof. Take $E_{0} \in \mathscr{F}(X)$ and $\varepsilon>0$. By (6.1) there exists an open covering $\left\{A_{i}\right\}$ of $E_{0}$ such that $\operatorname{diam}\left(A_{i}\right) \leq \delta$ for every $i$ and

$$
\sum_{i}\left(\operatorname{diam}\left(A_{i}\right)\right)^{d}<\mathscr{H}_{\delta}^{d}\left(E_{0}\right)+\varepsilon
$$

Since the union $A$ of all $A_{i}$ is open and the set $E_{0}$ is compact, then $A$ contains all sets $E$ in some neighbourhood of $E_{0}$, and $\mathscr{H}_{\delta}^{d}(E) \leq \mathscr{H}_{\delta}^{d}\left(E_{0}\right)+\varepsilon$ for all such $E$.

Proof of Proposition 6.1. We only prove the result for $E^{d}$; the proof for $E_{y}^{d}$ is essentially the same.

Let $\mathscr{G}$ be the class of all connected, closed sets $C$ in $X$ which are contained in $E_{y}$ for some $y \in Y$. It is easy to show that $\mathscr{G}$ is closed in $\mathscr{F}(X)$, and therefore

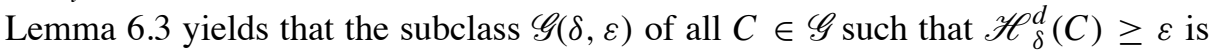
also closed in $\mathscr{F}(X)$ for every $\delta, \varepsilon>0$. Hence the union $G(\delta, \varepsilon)$ of all sets $C$ in $\mathscr{G}_{c}(\delta, \varepsilon)$ is closed in $X$ by Lemma 6.2.

To conclude the proof it suffices to verify that $E^{d}$ agrees with the union of all $G(1 / m, 1 / n)$ with $m, n=1,2, \ldots$ 


\section{Appendix: a density lemma}

In this appendix we give a density result used in the proof of Lemma 2.14.

7.1. Notation. Let $I$ be the interval $[0,1]$. As usual, $I^{*}$ is the metric space obtained by identifying the end points of $I$ (see Subsection 2.2), and $\operatorname{Lip}(I)$ stands for the Banach space of Lipschitz functions $\varphi: I \rightarrow \mathbb{R}$ endowed with the norm

$$
\|\varphi\|:=\|\varphi\|_{\infty}+\|\dot{\varphi}\|_{\infty} ;
$$

we denote by $\operatorname{Lip}_{*}(I)$ and $\operatorname{Lip}_{0}(I)$ the subspaces of all functions $\varphi \in \operatorname{Lip}(I)$ such that $\varphi(0)=\varphi(1)$, and $\varphi(0)=\varphi(1)=0$, respectively (thus $\operatorname{Lip}_{*}(I)$ agrees with the space of Lipschitz functions on $\left.I^{*}\right)$.

Given a Lipschitz map $\gamma: I \rightarrow \mathbb{R}^{d}$, we denote by $X(\gamma)$ the subspace of all $\varphi \in \operatorname{Lip}(I)$ of the form $\varphi=f \circ \gamma$ where $f$ is a smooth function with compact support on $\mathbb{R}^{d}$, and by $X_{*}(\gamma)$ and $X_{0}(\gamma)$ the intersections of $X(\gamma)$ with $\operatorname{Lip}_{*}(I)$ and $\operatorname{Lip}_{0}(I)$, respectively.

Finally, we denote by $\tau$ the topology on $\operatorname{Lip}(I)$ induced by the class $\mathscr{T}$ of all bounded linear functional $\Lambda$ on $\operatorname{Lip}(I)$ of the form

$$
\langle\Lambda ; \varphi\rangle:=\int_{I} \dot{\varphi} a d \mathscr{L}^{1}+\int_{I} \varphi d \mu,
$$

where $a$ is a function in $L^{1}(I)$ and $\mu$ a real-valued measure on $I$.

Proposition 7.2. The space $X(\gamma)$ [respectively $X_{*}(\gamma), X_{0}(\gamma)$ ] is $\tau$-dense in $\operatorname{Lip}(I)\left[\right.$ respectively $\left.\operatorname{Lip}_{*}(I), \operatorname{Lip}_{0}(I)\right]$ if and only if the following conditions hold:

(i) $\gamma$ is injective on $I$ [respectively $\left.I^{*}\right]$;

(ii) $\dot{\gamma} \neq 0$ a.e. in $I$.

Remark 7.3. (i) The topology $\tau$ makes $\operatorname{Lip}(I)$ a separated, locally convex topological vector space. The class $\mathscr{T}$ is strictly contained in the dual of $\operatorname{Lip}(I)$, thus $\tau$ is coarser than the weak topology. More precisely, if we identify Lip $(I)$ with $\mathbb{R} \times L^{\infty}(I)$ via the isomorphism $\varphi \mapsto(\varphi(0), \dot{\varphi})$, then $\tau$ corresponds to the weak* topology of $\mathbb{R} \times L^{\infty}(I)$, viewed as dual of $\mathbb{R} \times L^{1}(I)$.

(ii) Note that a weak* dense subspace of $L^{\infty}(I)$ is not necessarily sequentially weak* dense, and therefore we do not know if assumptions (i) and (ii) in Proposition 7.2 imply that $X(\gamma)$ is sequentially $\tau$-dense in $\operatorname{Lip}(I)$, or, equivalently, that every function in $\operatorname{Lip}(I)$ is the limit of a sequence of uniformly Lipschitz functions in $X(\gamma)$.

Proposition 7.2 has the following straightforward corollary.

Corollary 7.4. Let $\Lambda$ be a functional of the form (7.1) such that $\langle\Lambda ; \varphi\rangle=0$ for all $\varphi$ in $X(\gamma)$. If $\gamma$ is injective on $I$ [respectively $\left.I^{*}\right]$ and $\dot{\gamma} \neq 0$ a.e., then $\langle\Lambda ; \varphi\rangle=0$ for all $\varphi$ in $\operatorname{Lip}(I)\left[\right.$ respectively $\left.\operatorname{Lip}_{*}(I)\right]$. 
Proof of Proposition 7.2. We prove the statement only for $\operatorname{Lip}_{0}(I)$; the remaining cases can be easily derived from this one (or proved essentially in the same way).

Recall that the $\tau$-density of $X_{0}(\gamma)$ in $\operatorname{Lip}_{0}(I)$ means that the following implication holds for every $\Lambda \in \mathscr{T}$ :

$$
\langle\Lambda ; \varphi\rangle=0 \forall \varphi \in X_{0}(\gamma) \Rightarrow\langle\Lambda ; \varphi\rangle=0 \forall \varphi \in \operatorname{Lip}_{0}(I) .
$$

Step 1: necessity of (i). Assume by contradiction that $\gamma$ is not injective on $I^{*}$, that is, there exist two different points $t_{1}, t_{2} \in I$, not both equal to 0 and 1 , such that $\gamma\left(t_{1}\right)=\gamma\left(t_{2}\right)$. Then implication (7.2) fails for the functional $\Lambda$ given by $a:=0$ and $\mu:=\delta_{t_{1}}-\delta_{t_{2}}$.

Step 2: necessity of (ii). Assume by contradiction that there exists a subset $A$ of $I$ such that $0<|A|<1$ and $\dot{\gamma}=0$ a.e. on $A$. Then implication (7.2) fails for the functional $\Lambda$ given by $a:=1_{A}$ and $\mu:=0$. Indeed $(f \circ \gamma)^{\prime}=\nabla f \cdot \dot{\gamma}=0$ a.e. on $A$ for every $f \in C_{c}^{\infty}\left(\mathbb{R}^{d}\right)$, and therefore $\langle\Lambda ; \varphi\rangle=0$ for every $\varphi \in X_{0}(\gamma)$; on the other hand $\langle\Lambda ; \varphi\rangle \neq 0$ if $\varphi$ is the Lipschitz function defined by $\varphi(0):=0$ and $\dot{\varphi}:=1_{A}-m$ with $m:=\mathscr{L}^{1}(A)$ (note that $\varphi$ belongs to $\operatorname{Lip}_{0}(I)$ ).

Step 3: sufficiency of (i) and (ii). For every $\Lambda \in \mathscr{T}$ which does not vanish on $\operatorname{Lip}_{0}(I)$ we must construct a function $\varphi \in X_{0}(\gamma)$ such that $\langle\Lambda ; \varphi\rangle \neq 0$.

Integrating by parts the second integral in (7.1) we obtain that

$$
\langle\Lambda ; \varphi\rangle:=\int_{I} \dot{\varphi} \alpha d \mathscr{L}^{1} \quad \text { for every } \varphi \in \operatorname{Lip}_{0}(I)
$$

where $\alpha:=a-u$ and $u$ is a $B V$ function on $I$ whose distributional derivative agrees with $\mu$.

Therefore the fact that $\Lambda$ does not vanish on $\operatorname{Lip}_{0}(I)$ implies that the distributional derivative of $\alpha$ does not vanish, that is, $\alpha$ does not agree a.e. with a constant.

Step 4: idea for the construction of $\varphi$. Since $\alpha$ is not constant, we can find two disjoint intervals $I_{1}$ and $I_{2}$ in $I$ such that $\alpha$ is close (in the $L^{1}$ sense) to a constant $c_{i}$ on each $I_{i}$, and $c_{1} \neq c_{2}$. Then we set $\varphi:=f \circ \gamma$ where $f$ is a smooth function chosen in such a way that $\varphi=0$ on the connected component of $I^{*} \backslash\left(I_{1} \cup I_{2}\right)$ which contains 0 and $\varphi=1$ on the other. Hence $\dot{\varphi}$ vanishes outside the intervals $I_{1}$ and $I_{2}$, has integral equal to 1 on one of them (say $I_{1}$ ), and equal to -1 on the other, and using that $\alpha \simeq c_{i}$ on $I_{i}$ we obtain $\langle\Lambda ; \varphi\rangle=\int_{I} \alpha \dot{\varphi} \simeq c_{1}-c_{2} \neq 0$.

Step 5: construction of $\varphi$. We write $I(t, \delta)$ for the interval $[t-\delta, t+\delta]$. Since the function $\alpha$ is not a.e. equal to a constant and $\dot{\gamma} \neq 0$ a.e. (assumption (ii)), we can find $t_{1}, t_{2} \in I$ such that

(a) $0<t_{1}<t_{2}<1$;

(b) $\alpha$ is approximately continuous at $t_{1}$ and $t_{2}$ and $\alpha\left(t_{1}\right) \neq \alpha\left(t_{2}\right)$;

(c) $\gamma$ is differentiable at $t_{1}$ and $t_{2}$ and $\dot{\gamma}\left(t_{1}\right), \dot{\gamma}\left(t_{2}\right) \neq 0$. 
Let us fix for the time being $\varepsilon>0$. Using (a), (b), and (c) we find $\delta_{0}=\delta_{0}(\varepsilon)>0$ such that

(d) $I\left(t_{1}, \delta_{0}\right)$ and $I\left(t_{2}, \delta_{0}\right)$ are disjoint and contained in $(0,1)$;

(e) $\int_{I\left(t_{i}, \delta\right)}\left|\alpha(t)-\alpha\left(t_{i}\right)\right| d t \leq \varepsilon \delta$ for every $\delta$ with $0<\delta \leq \delta_{0}$ and $i=1,2$;

(f) $\left|\gamma(t)-\gamma\left(t_{i}\right)-\dot{\gamma}\left(t_{i}\right)\left(t-t_{i}\right)\right| \leq \varepsilon\left|t-t_{i}\right|$ for $t \in I\left(t_{i}, \delta_{0}\right)$ and $i=1,2$.

Let us fix for the time being $\delta$ with $0<\delta \leq \delta_{0}$, and denote by $\rho=\rho(\delta)$ the distance between the compact sets

$$
C_{0}:=\gamma\left(\left[0, t_{1}-\delta\right] \cup\left[t_{2}+\delta, 1\right]\right) \quad \text { and } \quad C_{1}:=\gamma\left(\left[t_{1}+\delta, t_{2}-\delta\right]\right) .
$$

Since $\gamma$ is injective $C_{0}$ and $C_{1}$ are disjoint, hence $\rho>0$ and therefore there exists a smooth function $f: \mathbb{R}^{d} \rightarrow[0,1]$ with compact support such that

(g) $f=0$ on $C_{0}$ and $f=1$ on $C_{1}$;

(h) $\|\nabla f\|_{\infty} \leq 2 / \rho .^{29}$

Finally we set $\varphi:=f \circ \gamma$. Since $\gamma(0)$ and $\gamma(1)$ belong to $C_{0}$, property (g) implies $\varphi(0)=\varphi(1)=0$, and therefore $\varphi$ belongs to $X_{0}(\gamma)$.

Step 6: estimate for $\langle\Lambda ; \varphi\rangle$. Property (g) implies that $\varphi$ is equal to 1 on $\left[t_{1}+\delta, t_{2}-\right.$ $\delta]$, and to 0 on $\left[0, t_{1}-\delta\right]$ and $\left[t_{2}+\delta, 1\right]$. Hence $\dot{\varphi}$ vanishes a.e. outside the intervals $I\left(t_{1}, \delta\right)$ and $I\left(t_{2}, \delta\right)$, and then

$$
\begin{aligned}
\langle\Lambda ; \varphi\rangle & =\sum_{i=1,2} \int_{I\left(t_{i}, \delta\right)} \dot{\varphi}(t) \alpha(t) d t \\
& =\sum_{i=1,2}\left[\int_{I\left(t_{i}, \delta\right)} \dot{\varphi}(t) \alpha\left(t_{i}\right) d t+\int_{I\left(t_{i}, \delta\right)} \dot{\varphi}(t)\left(\alpha(t)-\alpha\left(t_{i}\right)\right) d t\right] \\
& =\sum_{i=1,2}\left[\alpha\left(t_{i}\right)\left(\varphi\left(t_{i}+\delta\right)-\varphi\left(t_{i}-\delta\right)\right)+\int_{I\left(t_{i}, \delta\right)} \dot{\varphi}(t)\left(\alpha(t)-\alpha\left(t_{i}\right)\right) d t\right] \\
& =\alpha\left(t_{1}\right)-\alpha\left(t_{2}\right)+\sum_{i=1,2} \int_{I\left(t_{i}, \delta\right)} \dot{\varphi}(t)\left(\alpha(t)-\alpha\left(t_{i}\right)\right) d t
\end{aligned}
$$

Setting $c:=\alpha\left(t_{1}\right)-\alpha\left(t_{2}\right)$ and taking into account (e), (h) we thus obtain

$$
\begin{aligned}
|\langle\Lambda ; \varphi\rangle-c| & \leq \sum_{i=1,2} \int_{I\left(t_{i}, \delta\right)}|\dot{\varphi}(t)|\left|\alpha(t)-\alpha\left(t_{i}\right)\right| d t \\
& \leq 2\|\dot{\varphi}\|_{\infty} \varepsilon \delta \leq 2\|\nabla f\|_{\infty}\|\dot{\gamma}\|_{\infty} \varepsilon \delta \leq 4\|\dot{\gamma}\|_{\infty} \frac{\varepsilon \delta}{\rho} .
\end{aligned}
$$

${ }^{29}$ For example, let $f$ be the function $\left(\left(3 / 2-2 \operatorname{dist}\left(x, C_{1}\right) / \rho\right) \wedge 1\right) \vee 0$ regularized by convolution with a smooth kernel with support contained in the ball with center 0 and radius $\rho / 4$. 
Step 7: estimate for $\rho$. Set $m:=\left|\dot{\gamma}\left(t_{1}\right)\right| \wedge\left|\dot{\gamma}\left(t_{2}\right)\right|$. Since the map $\gamma$ is injective on $I^{*}$ (assumption (i)), there exists $r=r\left(\delta_{0}\right)>0$ such that $\left|\gamma(t)-\gamma\left(t^{\prime}\right)\right| \geq r$ whenever $\left|t-t^{\prime}\right| \geq \delta_{0}$ and $1-\left|t-t^{\prime}\right| \geq \delta_{0}$.

We claim that

$$
\rho \geq r \wedge 2(m-\varepsilon) \delta
$$

By the definition of $\rho$ this means that for every $t \in\left[0, t_{1}-\delta\right] \cup\left[t_{2}+\delta, 1\right]$ and $t^{\prime} \in\left[t_{1}+\delta, t_{2}-\delta\right]$ there holds $\left|\gamma(t)-\gamma\left(t^{\prime}\right)\right| \geq r \wedge 2(m-\varepsilon) \delta$.

Assume indeed that $\left|\gamma(t)-\gamma\left(t^{\prime}\right)\right|<r$. Then the definition of $r$ and property (d) imply that $t, t^{\prime} \in I\left(t_{i}, \delta_{0}\right)$ either for $i=1$ or $i=2$, and then property (f) yields

$$
\begin{aligned}
\left|\gamma(t)-\gamma\left(t^{\prime}\right)\right| & \geq\left|\dot{\gamma}\left(t_{i}\right)\right|\left|t-t^{\prime}\right|-\varepsilon\left|t-t_{i}\right|-\varepsilon\left|t^{\prime}-t_{i}\right| \\
& \geq(m-\varepsilon)\left|t-t^{\prime}\right| \geq 2(m-\varepsilon) \delta,
\end{aligned}
$$

where we have used that $\left|t-t^{\prime}\right|=\left|t-t_{i}\right|+\left|t^{\prime}-t_{i}\right|$ (note that $t_{i}$ belongs to the interval with end points $\left.t, t^{\prime}\right)$.

Step 8: conclusion. Since $\varepsilon, m, r$ do not depend on $\delta$, we can choose the latter so that $2(m-\varepsilon) \delta \leq r$. Then estimates (7.4) and (7.5) yield

$$
|\langle\Lambda ; \varphi\rangle-c| \leq 2\|\dot{\gamma}\|_{\infty} \frac{\varepsilon}{m-\varepsilon},
$$

and to obtain that $\langle\Lambda ; \varphi\rangle \neq 0$ it suffices to choose $\varepsilon$ so that the right-hand side of the previous inequality is strictly smaller than $|c|$ (such $\varepsilon$ exists because $m$ does not depend on $\varepsilon$ and $c$ does not vanish).

\section{Appendix: A proof of the triod lemma}

In this appendix we give a self-contained proof of Lemma 2.15.

Lemma 8.1. Let $D$ be a closed disc in $\mathbb{R}^{2}$, and let $C_{1}, C_{2}$ be curves parametrized by $\gamma_{1}, \gamma_{2}:[0,1] \rightarrow D$ with end points $\gamma_{i}(0), \gamma_{i}(1)$ in $\partial D$ for $i=1,2$. If $C_{1}$ and $C_{2}$ are disjoint, then $\gamma_{2}(0)$ and $\gamma_{2}(1)$ belong to the same connected component of $\partial D \backslash\left\{\gamma_{1}(0), \gamma_{1}(1)\right\}$.

Proof. Assume by contradiction that $\gamma_{2}(0)$ belongs to one of the two arcs of the circle $\partial D$ with end points $\gamma_{1}(0)$ and $\gamma_{1}(1)$ while $\gamma_{2}(1)$ belongs to the other. Then we can extend both paths $\gamma_{1}, \gamma_{2}$ to $[-1,1]^{*}$ so that the extensions are smooth on $[-1,0]$, map the interval $(-1,0)$ in the complement of $D$, and there exists only one couple $\left(t_{1}, t_{2}\right)$ such that $\gamma_{1}\left(t_{1}\right)=\gamma_{2}\left(t_{2}\right)$, and in that case the vectors $\dot{\gamma}_{1}\left(t_{1}\right)$ and $\dot{\gamma}_{2}\left(t_{2}\right)$ are linearly independent (see Figure 8.1).

Then $\Gamma\left(s_{1}, s_{2}\right):=\gamma_{1}\left(s_{1}\right)-\gamma_{2}\left(s_{2}\right)$ is a map from the torus $[-1,1]^{*} \times[-1,1]^{*}$ to $\mathbb{R}^{2}$, and the assumptions on $\gamma_{1}$ and $\gamma_{2}$ imply that $\Gamma^{-1}(0)$ contains only one point, and this point is regular. Hence the Brower degree of $\Gamma$ must be \pm 1 . On the other hand $\Gamma$ is not surjective on $\mathbb{R}^{2}$ because its domain is compact, and therefore its Brower degree must be 0 , hereby a contradiction. 


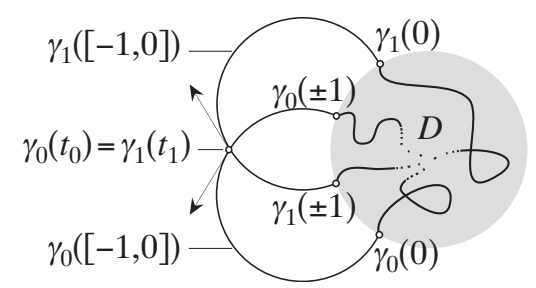

Figure 8.1. Proof of Lemma 8.1: extension of paths $\gamma_{1}$ and $\gamma_{2}$.

Corollary 8.2. Let $D$ be a closed disc in $\mathbb{R}^{2}$, and let $E_{1}$ and $E_{2}$ be close subsets of $D$ which are connected by curves. If $E_{1}$ and $E_{2}$ are disjoint, then $E_{1} \cap \partial D$ is contained in one connected component of $\partial D \backslash E_{2}$.

Proof. Assume by contradiction that there exist $x_{1}, y_{1} \in E_{1} \cap \partial D$ which do not belong to the same connected component of $\partial D \backslash E_{2}$. Then the two $\operatorname{arcs}$ of $\partial D$ with end points $x_{1}$ and $y_{1}$ must both contain points of $E_{2}$, say $x_{2}$ and $y_{2}$. Thus $x_{2}$ and $y_{2}$ belongs to different connected components of $\partial D \backslash\left\{x_{1}, y_{1}\right\}$. But for $i=1,2$ there exist curves $C_{i}$ contained in $E_{i}$ and connecting $x_{i}$ and $y_{i}$, and this contradicts Lemma 8.1.

Proof of Lemma 2.15. Let $D$ be an open disc in $\mathbb{R}^{2}$ with center $x$ and radius $\rho$. For every triod $Y=C_{1} \cup C_{2} \cup C_{3}$ with center $y$ we set

$$
r:=\min _{i=1,2,3}\left\{\max _{z \in C_{i}}|z-y|\right\} \text {. }
$$

If $y \in D$ and $r \geq|x-y|+\rho$, then for every $i$ there exists $z \in C_{i}$ which does not belong to $D$, and therefore $C_{i}$ must intersect the circle $\partial D$. Then we denote by $t_{i}$ the smallest $t$ such that $\gamma_{i}(t) \in \partial D$ (where $\gamma_{i}$ are taken as in Subsection 2.3), and by $E(Y)$ the 'truncated' triod

$$
E(Y):=\bigcup_{i=1}^{3} \gamma_{i}\left(\left[a_{i}, t_{i}\right]\right)
$$

(see Figure 8.2(a)). Moreover we let $F(Y)$ be the set of end points of $E(Y)$, i.e.,

$$
F(Y):=\left\{\gamma_{i}\left(t_{i}\right): i=1,2,3\right\}=E(Y) \cap \partial D .
$$

Finally, let $\mathscr{F}_{D}$ denote the family of all $Y \in \mathscr{F}$ such that $y \in D$ and $r \geq|x-y|+\rho$, and $\mathscr{G}_{D}$ the family of all $F(Y)$ with $Y \in \mathscr{F}_{D}$.

Step 1 . The family $\mathscr{F}$ is the union of $\mathscr{F} D$ over all discs $D$ whose centers have rational coordinates, and whose radii are rational. Hence, to prove that $\mathscr{F}$ is countable it suffices to show that each $\mathscr{F}_{D}$ is countable.

Step 2. Since the elements of $\mathscr{F}_{D}$ are pairwise disjoint and $F(Y) \subset Y$, the map $Y \mapsto F(Y)$ is injective on $\mathscr{F}_{D}$. Therefore to prove that $\mathscr{F}_{D}$ is countable it suffices to show that $\mathscr{G}_{D}$ is countable. 
(a)

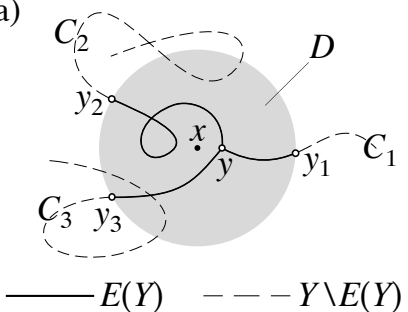

(b)

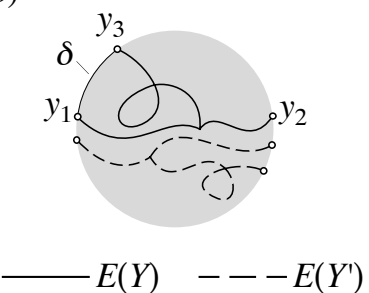

Figure 8.2. Proof of Lemma 2.15: (a) truncation of the triod $Y$; (b) the distance between $F(Y)$ and $F\left(Y^{\prime}\right)$ is larger than $\delta$.

Step 3. On the circle $\partial D$ we consider the geodesic distance $d,{ }^{30}$ and endow the set $\mathscr{G}_{D}$ with the Hausdorff distance $d_{H}$ associated to $d$ (see (2.1)). Since the metric space $\mathscr{G}_{D}$ is separable, to prove that it is countable it suffices to show that it contains only isolated points.

Step 4. Given $Y \in \mathscr{F}_{D}$, let $\delta$ be the length of the shortest arc in $\partial D$ with end points in $F(Y)=\left\{y_{1}, y_{2}, y_{3}\right\}$ (see Figure 8.2(b)). We claim that $d_{H}\left(F(Y), F\left(Y^{\prime}\right)\right) \geq \delta$ for every $Y^{\prime} \in \mathscr{F}_{D}$ with $Y^{\prime} \neq Y$. Indeed the triods $E(Y)$ and $E\left(Y^{\prime}\right)$ are disjoint subsets of the closure of $D$, and Corollary 8.2 implies that $E\left(Y^{\prime}\right) \cap \partial D=F\left(Y^{\prime}\right)$ is contained in one connected component of $\partial D \backslash E(Y)=\partial D \backslash F(Y)$, for example the arc with end points $y_{1}, y_{2}$ that does not contains $y_{3}$. Then the distance of $y_{3}$ from $F\left(Y^{\prime}\right)$ is at least $\delta$, which implies the claim.

\section{References}

[1] G. Alberti, S. BiAnChINI and G. CRIPPA, A uniqueness result for the continuity equation in two dimensions, J. Eur. Math. Soc. (JEMS), to appear. Available at http/cvgmt.sns.it/

[2] S. M. BATES, Toward a precise smoothness hypothesis in Sard's theorem, Proc. Amer. Math. Soc. 117 (1993), 279-283.

[3] B. Bojarski, P. HaJlasz and P. StRZelecki, Sard's theorem for mappings in Hölder and Sobolev spaces, Manuscripta Math. 118 (2005), 383-397.

[4] J. Bourgain, M. V. KorobKov and J. Kristensen, On the Morse-Sard property and level sets of Sobolev and BV functions, Rev. Mat. Iberoam. 29 (2013), 1-23.

[5] L. De PaSCALE, The Morse-Sard theorem in Sobolev spaces, Indiana Univ. Math. J. 50 (2001), 1371-1386.

[6] A.YA. Dubovicki1, On the structure of level sets of differentiable mappings of an $n$ dimensional cube into a $k$-dimensional cube (Russian), Izv. Akad. Nauk SSSR. Ser. Mat. 21 (1957), 371-408.

[7] R. Engelking, "General Topology", revised and completed edition, Sigma Series in Pure Mathematics, Vol. 6, Heldermann Verlag, Berlin, 1989.

[8] K. J. FAlconer, "The Geometry of Fractal Sets", Cambridge Tracts in Mathematics, Vol. 85, Cambridge University Press, Cambridge, 1985.

[9] H. FEDERER, "Geometric Measure Theory", Grundlehren der mathematischen Wissenschaften, Vol. 153, Springer, Berlin-New York 1969; reprinted in the series Classics in Mathematics, Springer, Berlin-Heidelberg, 1996.

30 That is, $d(x, y)$ is the length of the shortest $\operatorname{arcs}$ in $\partial D$ with end points $x, y$. 
[10] A. Figalli, A simple proof of the Morse-Sard theorem in Sobolev spaces, Proc. Amer. Math. Soc. 136 (2008), 3675-3681.

[11] E. L. GRINBERG, On the smoothness hypothesis in Sard's theorem, Amer. Math. Monthly 92 (1985), 733-734.

[12] M. W. HIRSCH, "Differential Topology", corrected reprint of the 1976 original, Graduate Texts in Mathematics, Vol. 33, Springer-Verlag, New York, 1976.

[13] S. V. Konyagin, On the level sets of Lipschitz functions, Tatra Mt. Math. Publ. 2 (1993), 51-59.

[14] S. G. Krantz and H. R. PARKs, "Geometric Integration Theory", Cornerstones, Birkhäuser, Boston, 2008.

[15] R. L. MOORE, Concerning triods in the plane and the junction points of plane continua, Proc. Nat. Acad. Sci. U.S.A. 14 (1928), 85-88.

[16] H. P. Mulholland, On the total variation of a function of two variables, Proc. London Math. Soc. (2) 46 (1940), 290-311.

[17] A. SARD, The measure of the critical values of differentiable maps, Bull. Amer. Math. Soc. 48 (1942), 883-890.

[18] L. Simon, "Lectures on Geometric Measure Theory", Proceedings of the Centre for Mathematical Analysis, Vol. 3, Australian National University, Centre for Mathematical Analysis, Canberra 1983.

[19] H. WhitNeY, A function not constant on a connected set of critical points, Duke Math. J. 1 (1935), 514-517.

Dipartimento di Matematica
Università di Pisa
largo Pontecorvo 5
56127 Pisa, Italy
galberti1@dm.unipi.it

S.I.S.S.A.

via Bonomea 265

34136 Trieste, Italy

bianchin@sissa.it

Departement Mathematik und Informatik Universität Basel

Rheinsprung 21

CH-4051 Basel, Switzerland

gianluca.crippa@unibas.ch 